\title{
INFRA-RED ARC SPECTRA PHOTOGRAPHED WITH XENOCYANINE
}

\author{
By W. F. Meggers and C. C. Kiess
}

\section{ABSTRACT}

A new sensitizing dye, xenocyanine, has made possible the preparation, by the Eastman Kodak Co., of plates highly sensitive to infra-red light ranging in wave length from 8,000 to $11,000 \mathrm{~A}$. With such plates the infra-red arc spectra of about 50 elements have been observed at the Bureau of Standards. New wavelength measurements have.been made for $\mathrm{Ti}, \mathrm{Fe}, \mathrm{Co}, \mathrm{Ni}$, and $\mathrm{Zr}$, and are presented in this paper. Many of these new lines have been accounted for as combinations between previously known terms of the neutral atoms. In the case of Ti a new term, $a^{5} \mathrm{D}$, has been found. Both $\mathrm{Ti}$ and $\mathrm{Fe}$ are rich in strong lines in the region investigated and are recommended as suitable comparison spectra for the measurement of wave lengths in the infra-red.

\section{CONTENTS}

I. Introduction

II. Apparatus and methods

III. Pesults

1. Titanium

2. Iron.

3. Cobalt

318

4. Nickel

5. Zirconium

IV. Conclusion

\section{INTRODUCTION}

More than a decade has passed since the authors ${ }^{1}$ published several papers on the infra-red emission spectra of various metallic arcs. Use was made of photographic plates bathed in solutions of dicyanin which was the best photographic sensitizer then known for the infrared. Dicyanin exhibits its maximum sensitivity in the red at wave length 7,000 A, but it is characterized by a broad band which made it possible to record, with prolonged exposures, the strongest lines between 9,000 and $10,000 \mathrm{~A}$. It appears now that the practical limit of dicyanin as employed by us is about $9,800 \mathrm{~A}$, and nearly all of the lines observed with apparently greater wave length have been explained as Lyman ghosts produced by the diffraction grating when very long exposures are used.

A new infra-red sensitizer, called neocyanine, ${ }^{2}$ was discovered in 1926; its sensitizing action is a maximum at 8,100 $\mathrm{A}$ and it has been used extensively for the photography of spectra. However, its sensitizing band is much narrower than that of dicyanin, the action falling off very rapidly beyond $9,000 \mathrm{~A}$, so that no appreciable advance was made in the study of infra-red spectra.

1 W. F. Meggers, B. S. Sei. Papers, vol. 14 (S312), p. 371, 1918. W. F. Meggers and C. C. Kiess, B. S, Sc.. Papers, vol. 14 (S524), p. 637, 1918. C. C. Kiess and W. H. Meggers, B. S. Sci. Papers, vol. Ju'(Sí72)

p. 2 . L. Dundon, A. L. Schoen, and R. M. Briggs, J. Opt. Soc. Am. and Rev. Sci. Inst., vol. 12, p. 397 , 1926. 
The recent discovery, in the Eastman Kodak research laboratories, of a new infra-red sensitizer, xenocyanine, is now recognized as beginning a new era in infra-red spectroscopy. Photographic emulsions containing xenocyanine have two remarkable properties-(1) a very broad sensitizing band which ranges from about 8,000 to $11,000 \mathrm{~A}$ with a maximum at 9,600 A, (2) extraordinary speed, which is estimated at one hundred to one thousand times that of the best materials heretofore available for the interval 9,000 to 10,000 A. At the Bureau of Standards more than 50 spectra have now been explored in the infrared with these new plates, and in nearly all cases many new lines have been discovered. In this paper results are presented for titanium, iron, cobalt, nickel, and zirconium.

\section{APPARATUS AND METHODS}

The light source in each case was a direct-current arc carrying 6 to 8 amperes with an applied potential of 220 volts. Rods of iron, cobalt, and nickel served as arc electrodes, while in the cases of titanium and zirconium, pieces of metal were used in a copper arc.

The spectrograms were made with a concave grating, mounted stigmatically, as described elsewhere, ${ }^{3}$ the dispersion being 10.4 $\mathrm{A} / \mathrm{mm}$ in the first-order spectrum. The infra-red spectra were photographed in the first order with exposures of 20 to 60 minutes and adjacent portions of the plate were exposed for 1 minute to the second-order spectrum of iron for the purpose of wave-length measurements. For titanium and iron the stronger lines were photographed also with the grating ruled with 20,000 lines per inch, giving a dispersion of $3.6 \mathrm{~A} / \mathrm{mm}$.

The xenocyanine plates were hypersensitized in a dilute ammonia bath just before use in the spectrograph, and they were developed in Eastman Kodak X-ray developer at about $18^{\circ} \mathrm{C}$. immediately after exposure.

Wave-length determinations in the infra-red spectra involved careful measurement of the position of the lines relative to lines in the blue and green portions of the iron spectrum recorded in juxtaposition. The values of the iron lines were those adopted as secondary standards by the International Astronomical Union, ${ }^{4}$ their apparent values for the interpolation of wave lengths corresponding to infrared lines being assumed to be exactly double their actual values.

\section{RESULTS}

\section{TITANIUM}

In Table 1 are presented the new wave lengths measured in the arc spectrum of Ti and extending it 1,000 A beyond the longest line previously recorded. The most complete list of $\mathrm{Ti}$ I lines published to date is that compiled by Prof. H. N. Russell ${ }^{5}$ from various sources and giving the classifications of nearly 1,400 lines as combinations between terms of the singlet, triplet, and quintet systems. Our list overlaps Russell's by nearly $1,500 \mathrm{~A}$, and a comparison of the two well illustrates the advantage of the new photographic materials over

${ }^{3}$ W. F. Meggers and K. Burns, B. S. Sci, Papers (No. 441), 18, p. 191, 1922.

Trans. Internat. Astron. Union, vol. 3, p. 77, 1928.

$\checkmark$ Astrophys. J., vol. 66, p. 347, 1927. 
those formerly used. Not only are the intensity estimates of the lines greatly increased but many faint lines, predictable from term combinations and heretofore unobserved, are now recorded.

In addition to wave length and intensity estimates, Table 1 records for each line its vacuum wave number and its term combination. Most of the classifications are based on the terms given by Russell which we have expressed in the current standard notation. ${ }^{6}$ A key for translating Russell's notation into that used at present is to be found in a recent paper by Miss Moore. ${ }^{7}$

As shown in Russell's analysis of Ti I a group of metastable terms is to be expected arising from the electron configuration $3 d^{4}$. Of this group of terms we have found $a^{5} \mathrm{D}$ which actually lies higher in the energy diagram than the lowest odd terms $\left(z^{5} \mathrm{D}^{\circ}\right.$ and $\left.z^{5} \mathrm{~F}^{\circ}\right)$ of the middle group with which it combines. The values of the components of $a^{5} \mathrm{D}$ relative to $a^{3} \mathrm{~F}_{2}=0$ are given in Table 2 .

TABLE 1.-Wave lengths in the infra-red spectrum of titanium

\begin{tabular}{|c|c|c|c|}
\hline$\lambda_{\mathrm{a} \text { ir I. A. }}$. & Intensity & $\nu_{\mathrm{VAC}} \mathrm{cm}^{-1}$ & Term combination \\
\hline $\begin{array}{l}10,774.82 \\
10,732.84 \\
10,731.11 \\
10,726.27 \\
10,689.33\end{array}$ & $\begin{array}{l}1 \\
1 \\
0 \\
4 \\
1\end{array}$ & $\begin{array}{l}9,278.36 \\
9,314.65 \\
9,316.15 \\
9,320.35 \\
9,352.56\end{array}$ & $\begin{array}{c}a^{5} \mathrm{~F}_{2}-z^{5} \mathrm{G}_{2}^{\circ} \\
a^{5} \mathrm{~F}_{3}-z^{5} \mathrm{G}_{3}^{\circ} \\
x^{5} \mathrm{D}_{3}^{\circ}-f^{5} \mathrm{~F}_{4} \\
a^{5} \mathrm{~F}_{1}-z^{5} \mathrm{G}_{2}^{\circ} \\
x^{5} \mathrm{D}_{4}^{\circ}-f^{5} \mathrm{~F}_{5}\end{array}$ \\
\hline $\begin{array}{l}10,676.90 \\
10,661.50 \\
10,607.71 \\
10,584.57 \\
10,552.87\end{array}$ & $\begin{array}{r}2 \\
8 \\
1 \\
12 \\
1\end{array}$ & $\begin{array}{l}9,363.44 \\
9,376.98 \\
9,424.52 \\
9,445.12 \\
9,473.50\end{array}$ & $\begin{array}{c}a^{5} \mathrm{~F}_{4}-z^{5} \mathrm{G}_{4}^{\circ} \\
a^{5} \mathrm{~F}_{2}-z^{5} \mathrm{G}_{3}^{\circ} \\
a^{5} \mathrm{~F}_{5}-z^{5} \mathrm{G}_{5}^{\circ} \\
a^{5} \mathrm{~F}_{3}-z^{5} \mathrm{G}_{4}^{\circ} \\
a^{3} \mathrm{H}_{5}-y^{3} \mathrm{G}_{4}^{\circ}\end{array}$ \\
\hline $\begin{array}{l}10,496.08 \\
10,459.98 \\
10,396.78 \\
10,145.40 \\
10,120.87\end{array}$ & $\begin{array}{r}15 \\
3 \\
20 \\
5 \\
7\end{array}$ & $\begin{array}{l}9,524.76 \\
9,557.63 \\
9,615.72 \\
9,853.98 \\
9,877.87\end{array}$ & $\begin{array}{r}a^{5} \mathrm{~F}_{4}-z^{5} \mathrm{G}_{5}^{\circ} \\
a^{3} \mathrm{H}_{6}-y^{3} \mathrm{G}_{5}^{\circ} \\
a^{5} \mathrm{~F}_{5}-z^{5} \mathrm{G}_{6}^{\circ} \\
a^{3} \mathrm{D}_{3}-x^{3} \mathrm{D}_{2}^{\circ}\end{array}$ \\
\hline $\begin{array}{l}10,066.47 \\
10,059.87 \\
10,057.69 \\
10,050.11 \\
10,048.78\end{array}$ & $\begin{array}{r}8 \\
12 \\
25 \\
5 \\
12\end{array}$ & $\begin{array}{l}9,931.25 \\
9,937.77 \\
9,939.92 \\
9,947.41 \\
9,948.73\end{array}$ & $\begin{array}{c}a^{3} \mathrm{D}_{2}-x^{3} \mathrm{D}_{1}^{\circ} \\
b^{3} \mathrm{~F}_{2}-z^{3} \mathrm{G}_{3}^{\circ} \\
a^{3} \mathrm{D}_{3}-x^{3} \mathrm{D}_{3}^{\circ} \\
\\
b^{3} \mathrm{~F}_{3}-z^{3} \mathrm{G}_{4}^{\circ}\end{array}$ \\
\hline $\begin{array}{r}10,034.45 \\
10,011.72 \\
10,003.02 \\
9,997.94 \\
9,981.16\end{array}$ & $\begin{array}{r}15 \\
15 \\
25 \\
15 \\
5\end{array}$ & $\begin{array}{r}9,962.94 \\
9,985.56 \\
9,994.24 \\
9,999.32 \\
10,016.13\end{array}$ & $\begin{array}{c}b^{3} \mathrm{~F}_{4}-z^{3} \mathrm{G}_{5}^{\circ} \\
a^{3} \mathrm{D}_{1}-x^{3} \mathrm{D}_{1}^{\circ} \\
a^{3} \mathrm{D}_{2}-x^{3} \mathrm{D}_{2}^{\circ} \\
a^{3} \mathrm{G}_{3}-y^{3} \mathrm{~F}_{2}^{\circ}\end{array}$ \\
\hline $\begin{array}{l}9,948.98 \\
9,941.33 \\
9,930.30 \\
9,927.35\end{array}$ & $\begin{array}{r}8 \\
8 \\
1 \\
20\end{array}$ & $\begin{array}{l}10,048.53 \\
10,056.26 \\
10,067.44 \\
10,070.42\end{array}$ & $\begin{array}{l}a^{3} \mathrm{D}_{1}-x^{3} \mathrm{D}_{2}^{\circ} \\
a^{3} \mathrm{D}_{2}-x^{3} \mathrm{D}_{3}^{\circ} \\
y^{1} \mathrm{G}_{4}^{\circ}-e^{1} \mathrm{G}_{4} \\
a^{3} \mathrm{G}_{4}-y^{3} \mathrm{~F}_{3}^{\circ}\end{array}$ \\
\hline $9,923.25$ & 2 & $10,074.58$ & $\left\{\begin{array}{l}a^{3} \mathrm{D}_{3}-y^{3} \mathrm{G}_{4}^{\circ} \\
y^{3} \mathrm{G}_{5}^{\circ}-e^{3} \mathrm{~F}_{4}\end{array}\right.$ \\
\hline
\end{tabular}

6 Phys. Rev., vol. 33, p. 900, 1929.

7 Astrophys. J., vol. 75, p. 235, 1932. 
TABLE 1.-Wave lengths in the infra-red spectrum of titanium-Continued

\begin{tabular}{|c|c|c|c|}
\hline$\lambda_{\text {air I. A. }}$. & Intensity & $\nu_{\mathrm{VRC}} \mathrm{cm}^{-1}$ & Term combination \\
\hline $\begin{array}{l}9,879.41 \\
9,832.15 \\
9,813.45 \\
9,787.67 \\
9,783.59\end{array}$ & $\begin{array}{r}3 \\
25 \\
5 \\
50 \\
20\end{array}$ & $\begin{array}{l}10,119.29 \\
10,167.93 \\
10,187.30 \\
10,214.14 \\
10,218.40\end{array}$ & $\begin{array}{l}a^{3} \mathrm{G}_{3}-y^{3} \mathrm{~F}_{3}^{\circ} \\
a^{3} \mathrm{G}_{3}^{-}-y^{3} \mathrm{~F}_{4}^{\circ} \\
z^{5} \mathrm{D}_{4}^{\circ}-a^{5} \mathrm{D}_{3} \\
a^{5} \mathrm{~F}_{3}-z^{5} \mathrm{~F}_{2}^{\circ} \\
a^{5} \mathrm{~F}_{2}-z^{3} \mathrm{~F}_{1}^{\circ}\end{array}$ \\
\hline $\begin{array}{l}9,783.30 \\
9,770.28 \\
9,768.22 \\
9,746.86 \\
9,743.60\end{array}$ & $\begin{array}{r}40 \\
40 \\
5 \\
15 \\
50\end{array}$ & $\begin{array}{l}10,218.70 \\
10,232.32 \\
10,234.47 \\
10,256.91 \\
10,260.34\end{array}$ & $\begin{array}{l}a^{5} \mathrm{~F}_{4}-z^{5} \mathrm{~F}_{3}^{\circ} \\
a^{5} \mathrm{~F}_{5}-z^{5} \mathrm{~F}_{4}^{\circ} \\
z^{5} \mathrm{D}_{3}^{\circ}-a^{5} \mathrm{D}_{2} \\
z^{5} \mathrm{D}_{4}^{\circ}-a^{5} \mathrm{D}_{4} \\
a^{5} \mathrm{~F}_{1}-z^{5} \mathrm{~F}_{1}^{\circ}\end{array}$ \\
\hline $\begin{array}{l}9,737.77 \\
9,728.36 \\
9,718.96 \\
9,717.00 \\
9,715.51\end{array}$ & $\begin{array}{r}5 \\
60 \\
25 \\
10 \\
3\end{array}$ & $\begin{array}{l}10,266.48 \\
10,276.41 \\
10,286.35 \\
10,288.42 \\
10,290.00\end{array}$ & $\begin{array}{l}z^{5} \mathrm{D}_{2}^{\circ}-a^{5} \mathrm{D}_{1} \\
a^{5} \mathrm{~F}_{2}-z^{5} \mathrm{~F}_{2}^{\circ} \\
a^{1} \mathrm{G}_{4}-z^{1} \mathrm{~F}_{3}^{\circ} \\
z^{5} \mathrm{D}_{3}^{\circ}-a^{5} \mathrm{D}_{3} \\
z^{5} \mathrm{D}_{1}^{\circ}-a^{5} \mathrm{D}_{0}\end{array}$ \\
\hline $\begin{array}{l}9,705.64 \\
9,702.86 \\
9,690.62 \\
9,688.86 \\
9,678.98\end{array}$ & $\begin{array}{r}80 \\
3 \\
2 \\
30 \\
3\end{array}$ & $\begin{array}{l}10,300.47 \\
10,303.42 \\
10,316.43 \\
10,318.31 \\
10,328.85\end{array}$ & $\begin{array}{l}a^{5} \mathrm{~F}_{3}-z^{5} \mathrm{~F}_{3}^{\circ} \\
z^{5} \mathrm{D}_{2}^{\circ}-a^{5} \mathrm{D}_{2} \\
a^{3} \mathrm{D}_{2}-z^{5} \mathrm{P}_{2}^{\circ} \\
a^{5} \mathrm{~F}_{1}-z^{5} \mathrm{~F}_{2}^{\circ} \\
z^{5} \mathrm{D}_{0}^{\circ}-a^{5} \mathrm{D}_{1}\end{array}$ \\
\hline $\begin{array}{l}9,675.55 \\
9,663.19 \\
9,661.42 \\
9,652.38 \\
9,651.66\end{array}$ & $\begin{array}{r}90 \\
3 \\
10 \\
1 \\
1\end{array}$ & $\begin{array}{l}10,332.50 \\
10,345.72 \\
10,347.61 \\
10,357.30 \\
10,358.07\end{array}$ & $\begin{array}{l}a^{5} \mathrm{~F}_{4}-z^{5} \mathrm{~F}_{4}^{\circ} \\
z^{5} \mathrm{D}_{1}^{\circ}-a^{5} \mathrm{D}_{2} \\
a^{3} \mathrm{D}_{3}-z^{5} \mathrm{P}_{3}^{\circ} \\
z^{5} \mathrm{D}_{2}^{\circ}-a^{5} \mathrm{D}_{3} \\
z^{5} \mathrm{D}_{3}^{\circ}-a^{5} \mathrm{D}_{4}\end{array}$ \\
\hline $\begin{array}{l}9,647.91 \\
9,647.40 \\
9,638.28 \\
9,606.77 \\
9,602.38\end{array}$ & $\begin{array}{r}1 \\
50 \\
100 \\
3 \\
1\end{array}$ & $\begin{array}{l}10,362.10 \\
10,362.65 \\
10,372.45 \\
10,406.48 \\
10,411.23\end{array}$ & $\begin{array}{c}a^{5} \mathbf{F}_{2}-z^{5} \mathbf{F}_{3}^{\circ} \\
a^{5} \mathbf{F}_{5}-z^{5} \mathbf{F}_{5}^{\circ} \\
y^{5} \mathbf{F}_{5}^{\circ}-f^{5} \mathbf{F}_{4} \\
y^{5} D_{3}^{0}-e^{5} \mathbf{F}_{4}\end{array}$ \\
\hline $\begin{array}{l}9,599.53 \\
9,590.15 \\
9,588.77 \\
9,570.08 \\
9,550.11\end{array}$ & $\begin{array}{r}50 \\
3 \\
4 \\
4 \\
2\end{array}$ & $\begin{array}{l}10,414.32 \\
10,424.51 \\
10,426.01 \\
10,446.37 \\
10,468.21\end{array}$ & $\begin{array}{l}a^{5} \mathrm{~F}_{3}-z^{5} \mathrm{~F}_{4}^{0} \\
y^{5} \mathrm{D}_{4}^{\circ}-e^{5} \mathrm{~F}_{5} \\
y^{5} \mathrm{~F}_{4}^{\circ}-f^{5} \mathrm{~F}_{3} \\
y^{5} \mathrm{~F}_{3}^{\circ}-f^{5} \mathrm{~F}_{2} \\
y^{5} \mathrm{~F}_{2}^{0}-f^{5} \mathrm{~F}_{1}\end{array}$ \\
\hline $\begin{array}{l}9,546.07 \\
9,511.80 \\
9,511.55 \\
9,510.81 \\
9,508.49\end{array}$ & $\begin{array}{r}50 \\
8 \\
10 \\
12 \\
20\end{array}$ & $\begin{array}{l}10,472.64 \\
10,510.37 \\
10,510.65 \\
10,511.47 \\
10,514.03\end{array}$ & $\begin{array}{l}a^{5} \mathrm{~F}_{4}-z^{5} \mathrm{~F}_{5}^{\circ} \\
y^{5} \mathrm{~F}_{2}^{\circ}-f^{5} \mathrm{~F}_{2} \\
y^{5} \mathrm{~F}_{1}^{\circ}-f^{5} \mathrm{~F}_{1} \\
y^{5} \mathrm{~F}_{3}^{\circ}-f^{5} \mathrm{~F}_{3} \\
y^{5} \mathrm{~F}_{4}^{\circ}-f^{5} \mathrm{~F}_{4}\end{array}$ \\
\hline $\begin{array}{l}9,506.04 \\
9,473.51 \\
9,453.22 \\
9,431.77 \\
9,409.69\end{array}$ & $\begin{array}{r}25 \\
2 \\
3 \\
3 \\
2\end{array}$ & $\begin{array}{l}10,516.76 \\
10,552.86 \\
10,575.51 \\
10,599.56 \\
10,624.44\end{array}$ & $\begin{array}{l}y^{5} \mathrm{~F}_{5}^{\circ}-f^{5} \mathrm{~F}_{5} \\
y^{5} \mathrm{~F}_{1}^{\circ}-f^{5} \mathrm{~F}_{2} \\
y^{5} \mathrm{~F}_{2}^{\circ}-f^{5} \mathrm{~F}_{3} \\
y^{5} \mathrm{~F}_{2}^{\circ}-f^{5} \mathrm{~F}_{4} \\
y^{5} \mathrm{~F}_{4}^{\circ}-f^{5} \mathrm{~F}_{5}\end{array}$ \\
\hline $\begin{array}{l}9,312.48 \\
9,305.04 \\
9,285.04 \\
9,257.62 \\
9,246.14\end{array}$ & $\begin{array}{r}4 \\
1 \\
5 \\
7 \\
10\end{array}$ & $\begin{array}{l}10,735.34 \\
10,743.92 \\
10,767.06 \\
10,798.95 \\
10,812.36\end{array}$ & $\begin{array}{l}x^{3} \mathrm{~F}_{2}^{\circ}-e^{3} \mathrm{~F}_{2} \\
y^{3} \mathrm{~F}_{4}^{o}-e^{3} \mathrm{G}_{4} \\
x^{3} \mathrm{~F}_{3}^{o}-e^{3} \mathrm{~F}_{3} \\
x^{3} \mathrm{~F}_{4}^{0}-e^{3} \mathbf{F}_{4} \\
y^{3} \mathrm{~F}_{4}^{\circ}-e^{3} \mathrm{G}_{3}\end{array}$ \\
\hline
\end{tabular}


TABLE 1.-Wave lengths in the infra-red spectrum of titanium-Continued

\begin{tabular}{|c|c|c|c|}
\hline$\lambda_{\text {sir I. A. }}$ I. & Intensity & $\nu_{\mathrm{Fac}} \mathrm{cm}^{-1}$ & Term combination \\
\hline $\begin{array}{l}9,170.38 \\
9,167.53 \\
9,135.92 \\
9,123.14 \\
9,090.70\end{array}$ & $\begin{array}{r}2 \\
8 \\
2 \\
5 \\
25\end{array}$ & $\begin{array}{l}10,901.68 \\
10,905.07 \\
10,942.80 \\
10,958.13 \\
10,997.24\end{array}$ & $\begin{array}{l}v^{3} \mathrm{D}_{3}^{\circ}-g^{3} \mathrm{~F}_{4} \\
y^{3} \mathrm{~F}_{3}^{\circ}-e^{3} \mathrm{G}_{4} \\
c^{3} \mathrm{P}_{1}-w^{3} \mathrm{D}_{2}^{\circ} \\
y^{3} \mathrm{~F}_{2}^{\circ}-e^{3} \mathrm{G}_{3} \\
a^{5} \mathrm{P}_{3}-z^{5} \mathrm{~S}_{2}^{\circ}\end{array}$ \\
\hline $\begin{array}{l}9,087.67 \\
9,086.94 \\
9,027.32 \\
9,023.65 \\
8,989.44\end{array}$ & $\begin{array}{r}2 \\
2 \\
15 \\
2 \\
12\end{array}$ & $\begin{array}{l}11,000.90 \\
11,001.79 \\
11,074.45 \\
11,078.95 \\
11,121.11\end{array}$ & $\begin{array}{l}c^{3} \mathrm{P}_{2}-w^{3} \mathrm{D}_{3}^{\circ} \\
a^{5} \mathrm{P}_{3}-y^{3} \mathrm{~F}_{2}^{\circ} \\
a^{5} \mathrm{P}_{2}-z^{5} \mathrm{~S}_{2}^{\circ} \\
a^{5} \mathrm{P}_{2}-y^{3} \mathrm{~F}_{2}^{\circ} \\
a^{5} \mathrm{P}_{1}-z^{5} \mathrm{~S}_{2}^{\circ}\end{array}$ \\
\hline $\begin{array}{l}8,985.80 \\
8,863.09 \\
8,821.14 \\
8,819.39 \\
8,794.40\end{array}$ & $\begin{array}{r}2 \\
3 \\
12 \\
8 \\
8\end{array}$ & $\begin{array}{l}11,125.62 \\
11,279.64 \\
11,333.29 \\
11,335.54 \\
11,367.75\end{array}$ & $\begin{array}{l}a^{5} \mathrm{P}_{1}-y^{3} \mathrm{~F}_{2}^{\circ} \\
x^{3} \mathrm{G}_{3}^{\circ}-f^{3} \mathrm{G}_{3} \\
a^{5} \mathrm{P}_{3}-y^{3} \mathrm{D}_{2}^{\circ} \\
a^{3} \mathrm{P}_{2}-z^{3} \mathrm{D}_{1}^{\circ} \\
z^{1} \mathrm{H}_{5}^{\circ}-e^{1} \mathrm{G}_{4}\end{array}$ \\
\hline $\begin{array}{l}8,778.66 \\
8,766.64 \\
8,761.44 \\
8,737.31 \\
8,734.70\end{array}$ & $\begin{array}{r}30 \\
75 \\
15 \\
7 \\
75\end{array}$ & $\begin{array}{l}11,388.13 \\
11,403.75 \\
11,410.52 \\
11,442.03 \\
11,445.45\end{array}$ & $\begin{array}{l}a^{5} \mathrm{P}_{3}-z^{3} \mathrm{P}_{2}^{\circ} \\
a^{3} \mathrm{P}_{2}-z^{3} \mathrm{D}_{2}^{\circ} \\
a^{5} \mathrm{P}_{2}-y^{3} \mathrm{D}_{2}^{\circ} \\
x^{3} \mathrm{G}_{5}^{\circ}-f^{3} \mathrm{G}_{5} \\
a^{3} \mathrm{P}_{1}-z^{3} \mathrm{D}_{1}^{\circ}\end{array}$ \\
\hline $\begin{array}{l}8,725.76 \\
8,719.56 \\
8,692.34 \\
8,682.99 \\
8,675.38\end{array}$ & $\begin{array}{r}6 \\
30 \\
100 \\
125 \\
150\end{array}$ & $\begin{array}{l}11,457.18 \\
11,465.33 \\
11,501.23 \\
11,513.61 \\
11,523.71\end{array}$ & $\begin{array}{l}a^{5} \mathrm{P}_{1}-y^{3} \mathrm{D}_{2}^{\circ} \\
a^{5} \mathrm{P}_{2}-z^{3} \mathrm{P}_{2}^{\circ} \\
a^{3} \mathrm{P}_{0}-z^{3} \mathrm{D}_{1}^{\circ} \\
a^{3} \mathrm{P}_{1}-z^{3} \mathrm{D}_{2}^{\circ} \\
a^{3} \mathrm{P}_{2}-z^{3} D_{3}^{\circ}\end{array}$ \\
\hline $\begin{array}{l}8,641.47 \\
8,636.38 \\
8,629.33 \\
8,618.44 \\
8,618.14\end{array}$ & $\begin{array}{l}40 \\
18 \\
18 \\
20 \\
15\end{array}$ & $\begin{array}{l}11,568.93 \\
11,575.75 \\
11,585.20 \\
11,599.85 \\
11,600.25\end{array}$ & $b^{3} \mathrm{P}_{1}-w^{3} \mathrm{D}_{1}^{\circ}$ \\
\hline $8,612.91$ & 7 & $11,607.29$ & $a^{5} \mathrm{P}_{2}-y^{5} \mathrm{P}_{i}^{\circ}$ \\
\hline $8,600.98$ & 25 & $11,623.39$ & $b^{3} \mathrm{P}_{2}-w^{3} \mathrm{D}_{2}^{0}$ \\
\hline $\begin{array}{l}8,598.18 \\
8,578.40 \\
8,569.72\end{array}$ & $\begin{array}{l}60 \\
15 \\
50\end{array}$ & $\begin{array}{l}11,627.18 \\
11,653.99 \\
11,665.79\end{array}$ & $\begin{array}{l}b^{1} \mathrm{G}_{4}-x^{3} \mathrm{G}_{3}^{\circ} \\
a^{5} \mathrm{P}_{1}-y^{5} \mathrm{D}_{1}^{\circ} \\
b^{3} \mathrm{P}_{0}-w^{3} \mathrm{D}_{1}^{\circ}\end{array}$ \\
\hline $\begin{array}{l}8,565.45 \\
8,550.54 \\
8,548.07 \\
8,539.36 \\
8,531.36\end{array}$ & $\begin{array}{r}25 \\
25 \\
100 \\
60 \\
15\end{array}$ & $\begin{array}{l}11,671.61 \\
11,691.96 \\
11,695.34 \\
11,707.26 \\
11,718.25\end{array}$ & $\begin{array}{l}a^{5} \mathrm{P}_{2}-y^{5} \mathrm{D}_{2}^{\circ} \\
a^{5} \mathrm{P}_{3}-y^{5} \mathrm{D}_{3}^{\circ} \\
a^{3} \mathrm{G}_{3}-x^{3} \mathrm{~F}_{2}^{\circ} \\
b^{3} \mathrm{P}_{1}-w^{3} \mathrm{D}_{2}^{\circ} \\
a^{5} \mathrm{P}_{1}-y^{5} \mathrm{D}_{2}^{\circ}\end{array}$ \\
\hline $\begin{array}{l}8,526.36 \\
8,525.99 \\
8,518.37 \\
8,518.05\end{array}$ & $\begin{array}{r}8 \\
8 \\
100 \\
60\end{array}$ & $\begin{array}{l}11,725.11 \\
11,725.63 \\
11,736.11 \\
11,736.55\end{array}$ & $\begin{array}{l}a^{3} \mathrm{G}_{4}-x^{3} \mathrm{~F}_{3}^{\circ} \\
z^{5} \mathrm{~F}_{5}^{\circ}-a^{5} \mathrm{D}_{4}\end{array}$ \\
\hline $8,496.03$ & 60 & $11,766.97$ & $\left\{\begin{array}{l}b^{3} \mathrm{P}_{2}-w^{3} \mathrm{D}_{3}^{\circ} \\
a^{1} \mathrm{~F}_{3}-v^{1} \mathrm{~F}_{3}^{\circ}\end{array}\right.$ \\
\hline $\begin{array}{l}8,495.51 \\
8,494.42 \\
8,483.16 \\
8,468.46 \\
8,467.15\end{array}$ & $\begin{array}{r}15 \\
30 \\
25 \\
100 \\
75\end{array}$ & $\begin{array}{l}11,767.69 \\
11,769.20 \\
11,784.83 \\
11,805.28 \\
11,807.11\end{array}$ & $\begin{array}{l}a^{5} \mathrm{P}_{2}-y^{5} \mathrm{D}_{3}^{\circ} \\
a^{3} \mathrm{G}_{3}-x^{3} \mathrm{~F}_{3}^{\circ} \\
a^{3} \mathrm{G}_{5}-x^{3} \mathrm{~F}_{4}^{\circ} \\
z^{5} \mathrm{~F}_{4}^{\circ}-a^{5} \mathrm{D}_{3}\end{array}$ \\
\hline
\end{tabular}


TABLE 1.-Wave lengths in the infra-red spectrum of titanium-Continued

\begin{tabular}{|c|c|c|c|}
\hline$\lambda_{\text {air I. A. }}$ & Intensity & $\nu_{\mathrm{VBC}} \mathrm{cm}^{-1}$ & Term combination \\
\hline $\begin{array}{l}8,460.96 \\
8,457.10 \\
8,450.89 \\
8,442.98 \\
8,438.93\end{array}$ & $\begin{array}{r}7 \\
40 \\
75 \\
20 \\
75\end{array}$ & $\begin{array}{l}11,815.74 \\
11,821.14 \\
11,829.74 \\
11,840.91 \\
11,846.60\end{array}$ & $\begin{array}{c}a^{5} \mathrm{P}_{3}-y^{5} \mathrm{D}_{4}^{\circ} \\
a^{3} \mathrm{H}_{5}-x^{3} \mathrm{G}_{4}^{\circ} \\
b^{3} \mathrm{P}_{2}-x^{5} \mathrm{D}_{3}^{\circ} \\
a^{3} \mathrm{H}_{6}-x^{3} \mathrm{G}_{5}^{\circ}\end{array}$ \\
\hline $\begin{array}{l}8,435.68 \\
8,434.98 \\
8,426.50 \\
8,424.41\end{array}$ & $\begin{array}{r}300 \\
300 \\
200 \\
50\end{array}$ & $\begin{array}{l}11,851.15 \\
11,852.14 \\
11,864.07 \\
11,867.01\end{array}$ & $\begin{array}{l}a^{5} \mathrm{~F}_{4}-z^{5} \mathrm{D}_{3}^{\circ} \\
a^{5} \mathrm{~F}_{5}-z^{5} \mathrm{D}_{4}^{\circ} \\
a^{5} \mathrm{~F}_{3}-z^{5} \mathrm{D}_{2}^{\circ} \\
z^{5} \mathrm{~F}_{3}^{\circ}-a^{5} \mathrm{D}_{2}\end{array}$ \\
\hline $\begin{array}{l}8,423.10 \\
8,418.70 \\
8,417.54 \\
8,416.97\end{array}$ & $\begin{array}{l}20 \\
10 \\
25 \\
60\end{array}$ & $\begin{array}{l}11,868.86 \\
11,875.06 \\
11,876.70 \\
11,877.50\end{array}$ & $\begin{array}{c}a^{3} \mathrm{G}_{4}-x^{3} \mathrm{~F}_{4}^{\circ} \\
z^{5} \mathrm{~F}_{4}^{\circ}-a^{5} \mathrm{D}_{4} \\
a^{3} \mathrm{H}_{4}-x^{3} \mathrm{G}_{3}^{\circ}\end{array}$ \\
\hline $\begin{array}{l}8,412.36 \\
8,407.87 \\
8,402.54 \\
8,396.93\end{array}$ & $\begin{array}{r}150 \\
4 \\
5 \\
90\end{array}$ & $\begin{array}{l}11,884.01 \\
11,890.37 \\
11,897.90 \\
11,905.84\end{array}$ & $\begin{array}{l}a^{5} \mathrm{~F}_{2}-z^{5} \mathrm{D}_{1}^{\circ} \\
a^{3} \mathrm{H}_{5}-x^{3} \mathrm{G}_{5}^{\circ} \\
a^{5} \mathrm{~F}_{1}-z^{5} \mathrm{D}_{0}^{\circ}\end{array}$ \\
\hline $\begin{array}{l}8,389.48 \\
8,382.82 \\
8,382.54 \\
8,377.90\end{array}$ & $\begin{array}{r}25 \\
90 \\
100 \\
100\end{array}$ & $\begin{array}{l}11,916.42 \\
11,925.89 \\
11,926.29 \\
11,932.90\end{array}$ & $\begin{array}{l}z^{5} \mathrm{~F}_{2}^{\circ}-a^{5} \mathrm{D}_{1} \\
a^{5} \mathrm{~F}_{1}-z^{5} \mathrm{D}_{1}^{\circ} \\
a^{5} \mathrm{~F}_{2}-z^{5} \mathrm{D}_{2}^{\circ} \\
a^{5} \mathrm{~F}_{3}-z^{5} \mathrm{D}_{3}^{\circ}\end{array}$ \\
\hline
\end{tabular}

Table 2.-The term $a^{5} D$

\begin{tabular}{|c|c|c|}
\hline$j$ & $\nu$ & $\Delta \nu$ \\
\hline 4 & $28,952.10$ & \\
3 & $28,882.44$ & 69.66 \\
2 & $28,828.51$ & 53.93 \\
1 & $28,791.62$ & 36.89 \\
0 & $28,772.86$ & 18.76 \\
\hline
\end{tabular}

\section{IRON}

The arc spectrum of iron is of exceptional interest because it is the main source of internationally adopted secondary standards of wave length. Furthermore, it is the spectrum in which the largest number of lines have been classified on the basis of modern theories of spectral structure, more than 2,400 lines now being accounted for as combinations of established atomic energy levels characteristic of the neutral atoms of iron.

Extensive analyses of the arc sprectrum of iron have been published by Burns and Walters ${ }^{8}$ and by Catalán, ${ }^{9}$ the former being based on new measurements of wave lengths in the vacuum arc, while the latter uses all available data for the arc in air and presents the most complete list of energy levels. Since our observations were also made at atmospheric pressure, we are quoting term combinations from the latter paper in Table 3 below. The results for the infra-red arc spectrum of iron appearing in Table 3 include measured wave lengths, estimated relative intensities, computed wave numbers in

${ }^{8} \mathrm{~K}$. Burns and F. M. Walters, jr., Pub. Allegheny Obs., vol. 6, No. 11, 1930.

๑ M. A. Catalán, Anales Soc. Esp. Fis. y Quim., vol. 28, p. 1239, 1930. 
vacuum, and spectral term combinations. In the range covered by these photographic observations lines were observed radiometrically by Randall and Barker. ${ }^{10}$ Without disparaging the excellent quality of the radiometric results as such, a comparison, nevertheless, shows the enormous advantage of the photographic method for recording details and improving precision of the measurements.

TABLE 3.-Wave lengths in the infra-red spectrum of iron

\begin{tabular}{|c|c|c|c|}
\hline$\lambda_{\text {air I. I. A. }}$ & Intensity & $\nu_{\mathrm{VAO}} \mathrm{cm}^{-1}$ & Term combination \\
\hline $\begin{array}{l}10,863.4 \\
10,817.9 \\
10,783.0 \\
10,532.12 \\
10,469.55\end{array}$ & $\begin{array}{r}1 \\
1 \\
1 \\
10 \\
20\end{array}$ & $\begin{array}{l}9,202.68 \\
9,241.38 \\
9,271.36 \\
9,492.17 \\
9,548.89\end{array}$ & $b^{3} \mathrm{D}_{3}^{\prime}-\alpha^{5} \mathrm{~F}_{4}$ \\
\hline $\begin{array}{l}10,452.60 \\
10,423.55 \\
10,395.75 \\
10,353.7 \\
10,348.16\end{array}$ & $\begin{array}{l}5 \\
3 \\
8 \\
2 \mathrm{~h} \\
4 \mathrm{~h}\end{array}$ & $\begin{array}{l}9,564.38 \\
9,591.04 \\
9,616.68 \\
9,655.77 \\
9,660.91\end{array}$ & $\begin{array}{r}a^{5} \mathrm{P}_{3}-a^{3} \mathrm{~F}_{4}{ }^{\prime} \\
d^{5} \mathrm{D}_{1}^{\prime}-\beta^{3} \mathrm{D}_{2}\end{array}$ \\
\hline $\begin{array}{l}10,345.2 \\
10,340.77 \\
10,218.36 \\
10,216.42 \\
10,195.11\end{array}$ & $\begin{array}{r}1 \\
4 \\
3 \\
100 \\
2\end{array}$ & $\begin{array}{l}9,663.70 \\
9,667.81 \\
9,783.63 \\
9,785.48 \\
9,805.94\end{array}$ & $\begin{array}{c}a^{5} \mathrm{P}_{2}-a^{3} \mathrm{~F}_{3}{ }^{\prime} \\
c^{5} \mathrm{~F}_{4}^{\prime}-20 \mathrm{C}_{5} \\
b^{3} \mathrm{D}_{3}^{\prime}-\alpha^{3} \mathrm{~F}_{4} \\
a^{3} \mathrm{G}_{4}-a^{3} \mathrm{~F}_{3}^{\prime}\end{array}$ \\
\hline $\begin{array}{l}10,187.4 \\
10,167.4 \\
10,145.64 \\
10,142.82 \\
10,115.18\end{array}$ & $\begin{array}{r}1 \\
1 \\
80 \\
2 \\
1\end{array}$ & $\begin{array}{l}9,813.35 \\
9,832.64 \\
9,853.75 \\
9,856.49 \\
9,883.42\end{array}$ & $\begin{array}{c}a^{5} \mathrm{P}_{2}-a^{5} \mathrm{~F}_{2}{ }^{\prime} \\
b^{3} \mathrm{D}_{2}^{\prime}-\alpha^{3} \mathrm{~F}_{3} \\
c^{5} \mathrm{~F}_{3}^{\prime}-29 \mathrm{C}_{3}\end{array}$ \\
\hline $\begin{array}{r}10,113.86 \\
10,071.88 \\
10,065.09 \\
10,057.64 \\
9,996.85\end{array}$ & $\begin{array}{c}2 \\
2 \\
60 \\
3 \\
1 \mathrm{~h}\end{array}$ & $\begin{array}{r}9,884.71 \\
9,925.91 \\
9,932.61 \\
9,939.97 \\
10,000.41\end{array}$ & $\begin{array}{l}b^{3} \mathrm{D}_{1}^{\prime}-\alpha^{3} \mathrm{~F}_{2} \\
c^{5} \mathrm{~F}_{4}^{\prime}-24 \mathrm{C}_{3}\end{array}$ \\
\hline $\begin{array}{l}9,980.55 \\
9,977.52 \\
9,944.13 \\
9,917.93 \\
9,897.69\end{array}$ & $\begin{array}{l}2 \mathrm{~h} \\
1 \\
3 \mathrm{~h} \\
1 \mathrm{p} ? \\
1 \mathrm{p} ?\end{array}$ & $\begin{array}{l}10,016.74 \\
10,019.78 \\
10,053.43 \\
10,079.99 \\
10,100.59\end{array}$ & $c^{5} \mathrm{~F}_{3}{ }^{\prime}-35 \mathrm{C}_{3}$ \\
\hline $\begin{array}{l}9,889.11 \\
9,881.4 \\
9,868.09 \\
9,861.83 \\
9,839.38\end{array}$ & $\begin{array}{r}40 \\
1 \\
3 \\
30 \\
1\end{array}$ & $\begin{array}{l}10,109.36 \\
10,117.2 \\
10,130.90 \\
10,137.33 \\
10,160.46\end{array}$ & $\begin{array}{l}c^{5} \mathrm{~F}_{4}{ }^{\prime}-30 \mathrm{C}_{5} \\
c^{5} \mathrm{~F}_{4}{ }^{\prime}-46 \mathrm{C}_{3} \\
c^{5} \mathrm{~F}_{3}{ }^{\prime}-39 \mathrm{C}_{3}\end{array}$ \\
\hline $\begin{array}{l}9,834.04 \\
9,811.36 \\
9,800.42 \\
9,794.91 \\
9,786.62\end{array}$ & $\begin{array}{c}3 \mathrm{~h} \\
2 \\
20 \\
1 \\
2\end{array}$ & $\begin{array}{l}10,165.97 \\
10,189.47 \\
10,200.85 \\
10,206.59 \\
10,215.24\end{array}$ & $\begin{array}{l}c^{5} \mathrm{~F}_{5}^{\prime}-21 \mathrm{C}_{5,4} \\
c^{5} \mathrm{~F}_{2}^{\prime}-49 \mathrm{C}_{3} \\
b^{3} \mathrm{~F}_{3}^{\prime}-\alpha^{5} \mathrm{~F}_{4}\end{array}$ \\
\hline $\begin{array}{l}9,783.96 \\
9,763.91 \\
9,763.34 \\
9,753.15 \\
9,747.24\end{array}$ & $\begin{array}{r}3 \\
15 \\
15 \\
10 \\
2\end{array}$ & $\begin{array}{l}10,218.01 \\
10,238.99 \\
10,239.59 \\
10,250.29 \\
10,256.50\end{array}$ & $\begin{array}{l}c^{5} \mathrm{~F}_{5}^{\prime}-22 \mathrm{C}_{5} \\
c^{5} \mathrm{~F}_{4}^{\prime}-33 \mathrm{C}_{4} \\
c^{5} \mathrm{~F}_{1}^{\prime}-56 \mathrm{C}_{2} \\
b^{3} \mathrm{D}_{2}^{\prime}-\alpha^{3} \mathrm{~F}_{2}\end{array}$ \\
\hline
\end{tabular}

${ }^{10}$ H. M. Randall and E. F. Barker, Astrophys. J., vol. 49, p. 42, 1919. 
TABLE 3.-Wave lengths in the infra-red spectrum of iron-Continued

\begin{tabular}{|c|c|c|c|}
\hline$\lambda_{\mathrm{air}} \mathrm{I} . A$ & Intensity & $\nu_{\nabla B O} \mathrm{~cm}^{-1}$ & Term combination \\
\hline $9,738.73$ & 200 & $10,265.47$ & \multirow{4}{*}{$\begin{array}{l}c^{5} \mathrm{~F}_{5}{ }^{\prime}-23 \mathrm{C}_{6} \\
a^{5} \mathrm{~S}_{2}{ }^{\prime}-48 \mathrm{C}_{2} \\
a^{5} \mathrm{~S}_{2}{ }^{\prime}-49 \mathrm{C}_{3}\end{array}$} \\
\hline $9,699.70$ & $6 \mathrm{~h}$ & $10,306.77$ & \\
\hline $\begin{array}{l}9,693.69 \\
9.683 .57\end{array}$ & 1 & $\begin{array}{l}10,313.16 \\
10,323.94\end{array}$ & \\
\hline $9,676.42$ & 1 & $10,331.57$ & \\
\hline $9,673.16$ & $1 \mathrm{~h}$ & $10,335.05$ & \multirow{4}{*}{$\begin{array}{c}c^{5} \mathrm{~F}_{3}^{\prime}-47 \mathrm{C}_{4,3} \\
c^{5} \mathrm{~F}_{2}^{\prime}-56 \mathrm{C}_{2} \\
b^{3} \mathrm{D}_{3}^{\prime}-\alpha^{3} \mathrm{~F}_{3}\end{array}$} \\
\hline $9,666.59$ & 2 & $10,342.07$ & \\
\hline $\begin{array}{l}9,658.94 \\
9.657 .30\end{array}$ & $\begin{array}{l}3 \\
4\end{array}$ & $\begin{array}{l}10,350.26 \\
10.352 .03\end{array}$ & \\
\hline $9,653.18$ & 20 & $10,356.44$ & \\
\hline $9,637.55$ & 2 & $10,373.25$ & \multirow{3}{*}{$\begin{array}{l}c^{5} \mathrm{~F}_{3}{ }^{\prime}-49 \mathrm{C}_{3} \\
c^{5} \mathrm{~F}_{4}^{\prime}-39 \mathrm{C}_{3}\end{array}$} \\
\hline $9,634.22$ & 5 & $10,376.82$ & \\
\hline $\begin{array}{l}9,626.60 \\
9,602.07\end{array}$ & $\begin{array}{l}30 h \\
2\end{array}$ & $\begin{array}{l}10,385.00 \\
10,411.57\end{array}$ & \\
\hline $9,569.95$ & $40 \mathrm{~h}$ & $10,446.51$ & $c^{5} \mathrm{~F}_{5}^{\prime}-30 \mathrm{C}_{5}$ \\
\hline $9,556.56$ & 1 & $10,461.14$ & \multirow{4}{*}{$\begin{array}{l}c^{5} \mathrm{D}_{2}^{\prime}-29 \mathrm{C}_{3} \\
b^{5} \mathrm{G}_{6}^{\prime}-\beta^{5} \mathrm{~F}_{5} \\
c^{5} \mathrm{~F}_{3}^{\prime}-54 \mathrm{C}_{4} \\
c^{5} \mathrm{~F}_{4}^{\prime}-45 \mathrm{C}_{5} \\
b^{5} \mathrm{G}_{2}^{\prime}-\beta^{5} \mathrm{~F}_{3}\end{array}$} \\
\hline $9,550.90$ & 2 & $10,467.35$ & \\
\hline $\begin{array}{l}9,529.31 \\
9,527.7\end{array}$ & $\begin{array}{l}4 \mathrm{~h} \\
1\end{array}$ & $\begin{array}{l}10,491.06 \\
10,492.8\end{array}$ & \\
\hline $9,513.21$ & $10 \mathrm{~h}$ & $10,508.82$ & \\
\hline $9,462.97$ & 2 & $10,564.61$ & \multirow{5}{*}{$\begin{array}{l}c^{5} \mathrm{D}_{3}{ }^{\prime}-24 \mathrm{C}_{3} \\
c^{5} \mathrm{~F}_{1}^{\prime}-61 \mathrm{C}_{2} \\
c^{5} \mathrm{~F}_{5}^{\prime}-33 \mathrm{C}_{4} \\
c^{5} \mathrm{~F}_{2}^{\prime}{ }^{\prime}-60 \mathrm{C}_{3} \\
b^{3} \mathrm{~F}_{3}{ }^{\prime}-\alpha^{5} \mathrm{~F}_{3}\end{array}$} \\
\hline $9,454.24$ & $4 \mathrm{~h}$ & $10,574.37$ & \\
\hline $9,452.45$ & 2 & $10,576.37$ & \\
\hline $\begin{array}{l}9,443.98 \\
9437.91\end{array}$ & $10 \mathrm{~h}$ & $10,585.85$ & \\
\hline $9,436.91$ & & $10,592.00$ & \\
\hline $9,430.07$ & 4 & $10,601.47$ & \multirow[b]{2}{*}{$c^{5} \mathrm{~F}_{3}{ }^{\prime}-58 \mathrm{C}_{2}$} \\
\hline $9,414.14$ & $20 \mathrm{~h}$ & $10,619.41$ & \\
\hline $9,401.09$ & $10 \mathrm{~h}$ & $\begin{array}{l}10,024.0 \\
10,634.15\end{array}$ & \multirow{2}{*}{$c^{5} \mathrm{~F}_{4}^{\prime}-50 \mathrm{C}_{4}$} \\
\hline $9,394 . ' 71$ & $3 \mathrm{~h}$ & $10,641.37$ & \\
\hline $9,388.28$ & $3 \mathrm{~h}$ & $10,648.66$ & \multirow{4}{*}{$\begin{array}{c}b^{3} \mathrm{~F}_{4}-a^{3} \mathrm{~F}_{4}^{\prime} \\
c^{5} \mathrm{D}_{1}^{\prime}-43 \mathrm{C}_{2} \\
a^{3} \mathrm{P}_{2}-a^{5} \mathrm{P}_{3}^{\prime} \\
b^{3} \mathrm{~F}_{4}-a^{3} \mathrm{D}_{3}^{\prime}\end{array}$} \\
\hline $9,382.83$ & $3 \mathrm{~h}$ & $10,654.84$ & \\
\hline $9,372.84$ & 6 & $10,666.20$ & \\
\hline $\begin{array}{l}9,362.36 \\
9,359.37\end{array}$ & $\begin{array}{l}4 \\
3\end{array}$ & $\begin{array}{l}10,678.13 \\
10,681.55\end{array}$ & \\
\hline $9,350.52$ & 10 & $10,691.66$ & \multirow{4}{*}{$\begin{array}{l}b^{3} \mathrm{~F}_{4}^{\prime}-\alpha^{5} \mathrm{~F}_{4} \\
c^{5} \mathrm{~F}_{4}^{\prime}-\alpha^{3} \mathrm{D}_{3} \\
c^{5} \mathrm{~F}_{5}^{\prime}-38 \mathrm{C}_{6} \\
c^{5} \mathrm{D}_{3}^{\prime}-29 \mathrm{C}_{3} \\
c^{5} \mathrm{~F}_{4}^{\prime}-54 \mathrm{C}_{4}\end{array}$} \\
\hline $9,343.40$ & 3 & $10,699.80$ & \\
\hline $9,333.94$ & 2 & $10,710.65$ & \\
\hline $\begin{array}{l}9,318.13 \\
9,307.94\end{array}$ & $\begin{array}{l}3 \\
2\end{array}$ & $\begin{array}{l}10,728.82 \\
10,740.57\end{array}$ & \\
\hline $9,294.66$ & 2 & $10,755.92$ & \multirow{4}{*}{$\begin{array}{l}c^{5} \mathrm{~F}_{4}^{\prime}-\beta^{5} \mathrm{D}_{4} \\
c^{5} \mathrm{D}_{4}^{\prime}-21 \mathrm{C}_{5,4} \\
b^{3} \mathrm{~F}_{3}^{\prime}-\alpha^{3} \mathrm{~F}_{4} \\
b^{3} \mathrm{~F}_{3}-a^{3} \mathrm{D}_{2}^{\prime} \\
b^{5} \mathrm{~F}_{1}^{\prime}-\alpha^{5} D_{1}\end{array}$} \\
\hline $9,259.05$ & 15 & $10,797.29$ & \\
\hline $9,258.40$ & 20 & $10,798.04$ & \\
\hline $\begin{array}{l}9,246.54 \\
9,242.32\end{array}$ & $\begin{array}{l}2 \\
2\end{array}$ & $\begin{array}{l}10,811.89 \\
10,816 . \text { S2 }\end{array}$ & \\
\hline $9,233.2$ & 1 & $10,827.5$ & \multirow{4}{*}{$\begin{array}{c}b^{5} \mathrm{G}_{5}{ }^{\prime}-\alpha^{3} \mathrm{G}_{3} \\
c^{5} \mathrm{~F}_{5}^{\prime}-45 \mathrm{C}_{5} \\
c^{5} \mathrm{D}_{4}^{\prime}-22 \mathrm{C}_{5} \\
b^{3} \mathrm{P}_{1}-b^{5} \mathrm{D}_{2}^{\prime}\end{array}$} \\
\hline $9,217.54$ & $5 \mathrm{~h}$ & $10,845.91$ & \\
\hline $9,214.45$ & 6 & $10,849.54$ & \\
\hline $\begin{array}{l}9,210.02 \\
9,199.52\end{array}$ & $\begin{array}{l}6 \\
2 \mathrm{~h}\end{array}$ & $\begin{array}{l}10,854.76 \\
10,867.15\end{array}$ & \\
\hline
\end{tabular}


TABLE 3.-Wave lengths in the infra-red spectrum of iron-Continued

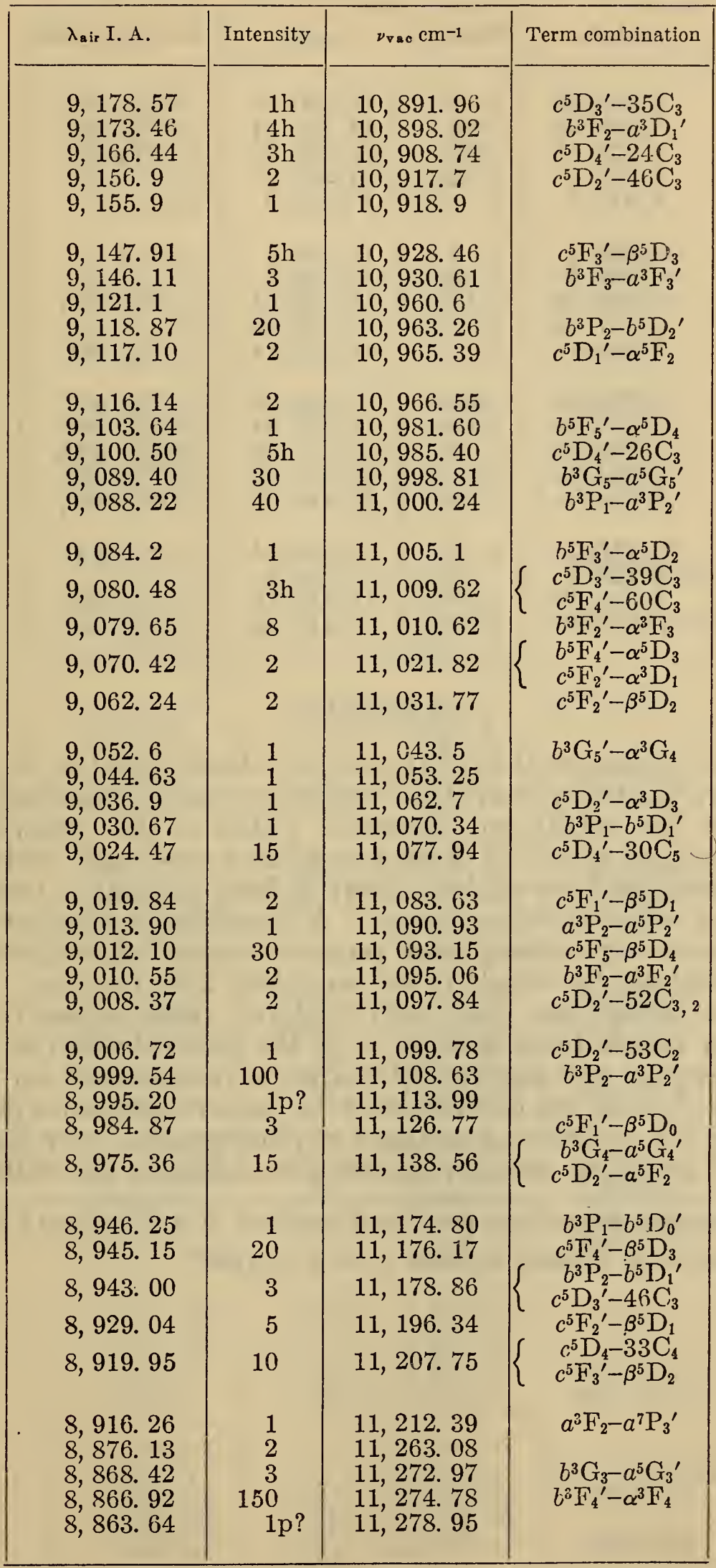


TABLE 3.-Wave lengths in the infra-red spectrum of iron-Continued

\begin{tabular}{|l|c|c|c|}
\hline$\lambda_{\text {sir }}$ I. A. & Intensity & $\nu_{\mathrm{vac}} \mathrm{cm}^{-1}$ & Term combination \\
\cline { 1 - 2 } $8,846.82$ & 5 & $11,300.40$ & \\
\hline $8,838.36$ & 30 & $11,311.21$ & $c^{5} \mathrm{D}_{1}{ }^{\prime}-61 \mathrm{C}_{2}$ \\
$8,824.18$ & 200 & $11,329.39$ & $b^{3} \mathrm{P}^{0}-a^{3} \mathrm{P}_{1} \mathrm{P}_{2}-a^{5} \mathrm{P}_{3}{ }^{\prime}$ \\
$8,814.5$ & 2 & $11,341.8$ & \\
$8,812.0$ & $1 \mathrm{p} ?$ & $11,345.0$ & \\
$8,809.1$ & 2 & $11,348.8$ & \\
$8,808.3$ & $4 \mathrm{~h}$ & $11,349.8$ & \\
$8,804.56$ & 10 & $11,354.64$ & $a^{3} \mathrm{P}_{2}-a^{5} \mathrm{P}_{1}{ }^{\prime}$ \\
$8,801.98$ & $1 \mathrm{p} ?$ & $11,357.96$ & \\
$8,796.42$ & 2 & $11,365.14$ & $c^{5} \mathrm{D}_{3}{ }^{\prime}-54 \mathrm{C}_{4}$ \\
& & & \\
$8,793.38$ & 120 & $11,369.07$ & $b^{3} \mathrm{~F}_{3}{ }^{\prime}-\alpha^{3} \mathrm{~F}_{3}$ \\
$8,790.62$ & $10 \mathrm{~h}$ & $11,372.64$ & $c^{5} \mathrm{D}_{2}{ }^{\prime}-60 \mathrm{C}_{3}$ \\
$8,784.44$ & 5 & $11,380.64$ & $c^{5} \mathrm{D}_{3}{ }^{\prime}-\beta^{5} \mathrm{D}_{4}$ \\
$8,764.02$ & 100 & $11,407.16$ & $b^{3} \mathrm{~F}_{2}{ }^{\prime}-\alpha^{3} \mathrm{~F}_{2}$ \\
$8,757.16$ & 50 & $11,416.09$ & $b^{3} \mathrm{P}_{1}-a^{3} \mathrm{P}_{1}{ }^{\prime}$ \\
$8,747.32$ & 2 & $11,428.94$ & \\
$8,729.1$ & 2 & $11,452.8$ & $b^{3} \mathrm{G}_{3}-a^{3} \mathrm{G}_{4}{ }^{\prime}$ \\
$8,713.19$ & 10 & $11,473.70$ & $b^{3} \mathrm{G}_{5}-a^{5} \mathrm{G}_{4}{ }^{\prime}$ \\
$8,710.29$ & $20 \mathrm{~h}$ & $11,477.53$ & $c^{5} \mathrm{D}_{4}-45 \mathrm{C}_{5}$ \\
\hline
\end{tabular}

\section{COBALT}

About 1,200 lines of the cobalt arc have been classified by Catalán and Bechert, ${ }^{11}$ but only 10 of these have wave lengths exceeding $8,648 \mathrm{~A}$. Our results for cobalt are shown in Table 4, the construction of which is identical with that of the preceding wave-length tables. The line classifications have all been derived from Catalán's term tables without any effort to extend these. A considerable number of lines, including some very strong ones, remain unclassified, indicating that the term analysis for cobalt is less complete than for iron. The arc spectrum of cobalt has also been explored radiometrically in the infra-red by Randall and Barker. ${ }^{12}$ In the interval 9,000 to $11,000 \mathrm{~A}$ the wave lengths and relative intensities were measured for 10 lines, all but two of which are identifiable with the strongest ones in our list. Here again the superior advantages of photographic over bolometric methods of studying complex spectra are strikingly exemplified.

11 M. A. Catalán and K. Bechert, Zeit.f. Phys., vol. 32, p. 336, 1925. M. A. Catalán, Zeit.f. Phys., vol. 47, p. 89,1927 .

${ }_{12}$ H. M. Randall and E. F. Barker, Astrophys. J., vol. 49, p. 54, 1919. 
TABLE 4.-Wave lengths in the infra-red spectrum of cobalt

\begin{tabular}{|c|c|c|c|}
\hline$\lambda_{\mathrm{Bir}}$ I. A. & Intensity & $\nu_{\mathrm{VaC}} \mathrm{cm}^{-1}$ & Term combination \\
\hline $\begin{array}{l}11,091.94 \\
10,805.97 \\
10,681.80 \\
10,660.15 \\
10,584.99\end{array}$ & $\begin{array}{l}2 \\
1 \\
4 \\
5 \\
2\end{array}$ & $\begin{array}{l}9,013.09 \\
9,251.61 \\
9,359.15 \\
9,378.16 \\
9,444.75\end{array}$ & $\begin{array}{c}b^{2} \mathrm{D}_{3}^{\prime}-\alpha^{4} \mathrm{~F}_{4} \\
c^{4} \mathrm{~F}_{4}^{\prime}-\left[{ }^{4} \mathrm{~F}_{5}\right]\end{array}$ \\
\hline $\begin{array}{l}10,521.36 \\
10,471.96 \\
10,447.40 \\
10,442.10 \\
10,398.37\end{array}$ & $\begin{array}{r}2 \\
10 \\
3 \\
15 \\
4\end{array}$ & $\begin{array}{l}9,501.87 \\
9,546.69 \\
9,569.14 \\
9,574.00 \\
9,614.26\end{array}$ & $\begin{array}{r}b^{2} \mathrm{D}_{2}-b^{4} \mathrm{D}_{3}{ }^{\prime} \\
b^{2} \mathrm{~F}_{3}{ }^{\prime}-\alpha^{4} \mathrm{~F}_{3} \\
b_{5}-\left[{ }^{4} \mathrm{~F}_{5}\right] \\
c^{4} \mathrm{G}_{6}{ }^{\prime}-\left[\mathrm{H}_{7}\right]\end{array}$ \\
\hline $\begin{array}{l}10,382.22 \\
10,374.65 \\
10,369.12 \\
10,367.95 \\
10,364.43\end{array}$ & $\begin{array}{r}30 \\
1 \\
2 \\
2 \\
1\end{array}$ & $\begin{array}{l}9,629.26 \\
9,636.24 \\
9,641.38 \\
9,642.47 \\
9,645.74\end{array}$ & $\begin{array}{l}b^{2} \mathrm{D}_{2}-a^{2} \mathrm{~F}_{3}{ }^{\prime} \\
c^{4} \mathrm{~F}_{3}{ }^{\prime}-\left[\mathrm{F}_{3}\right] \\
c^{4} \mathrm{G}_{6}{ }^{\prime}-\left[{ }^{4} \mathrm{~F}_{5}\right] ? \\
c^{4} \mathrm{~F}_{4}^{\prime}-\left[\mathrm{D}_{3}{ }^{\prime}\right] \\
c^{4} \mathrm{G}_{6}{ }^{\prime}-\left[\mathrm{H}_{6}\right]\end{array}$ \\
\hline $\begin{array}{l}10,354.45 \\
10,348.00 \\
10,335.40 \\
10,332.65 \\
10,327.32\end{array}$ & $\begin{array}{r}60 \\
1 \\
4 \\
3 \\
2\end{array}$ & $\begin{array}{l}9,655.04 \\
9,661.1 \\
9,672.84 \\
9,675.41 \\
9,680.40\end{array}$ & $\begin{array}{l}b^{2} \mathrm{~F}_{4}{ }^{\prime}-\alpha^{4} \mathrm{~F}_{4} \\
c^{4} \mathrm{~F}_{3}{ }^{\prime}-\left[\mathrm{F}_{4}, \mathrm{D}_{3}\right] \\
c^{4} \mathrm{G}_{6}{ }^{\prime}-\left[{ }^{4} \mathrm{G}_{6},{ }^{4} \mathrm{~F}_{5}\right]\end{array}$ \\
\hline $\begin{array}{l}10,276.80 \\
10,265.4 \\
10,244.64 \\
10,239.1 \\
10,222.12\end{array}$ & $\begin{array}{l}5 \mathrm{~h} \\
1 \mathrm{~h} \\
1 \\
1 \mathrm{~h} \\
1\end{array}$ & $\begin{array}{l}9,727.99 \\
9,738.8 \\
9,758.52 \\
9,763.8 \\
9,780.03\end{array}$ & $\begin{array}{r}\left(m^{2} \mathrm{~F}_{3}{ }^{\prime}\right)-\left[\mathrm{F}_{3}\right] \\
\\
{ }^{2} \mathrm{G}_{5}-{ }^{6} \mathrm{G}_{4}{ }^{\prime} \\
a^{2} \mathrm{D}_{2}-{ }^{6} \mathrm{D}_{1}^{\prime}\end{array}$ \\
\hline $\begin{array}{l}10,195.3 \\
10,172.83 \\
10,167.58 \\
10,154.90 \\
10,152.93\end{array}$ & $\begin{array}{c}1 \\
20 \mathrm{~h} \\
200 \\
2 \\
4\end{array}$ & $\begin{array}{l}9,805.75 \\
9,827.41 \\
9,832.49 \\
9,844.77 \\
9,846.68\end{array}$ & $\begin{array}{c}c^{4} \mathrm{~F}_{5}^{\prime}-\left[{ }^{4} \mathrm{D}_{4}\right] \\
b^{2} \mathrm{D}_{3}^{\prime}-\alpha^{2} \mathrm{~F}_{2}\end{array}$ \\
\hline $\begin{array}{l}10,131.38 \\
10,128.05 \\
10,111.1 \\
10,105.40 \\
10,092.0\end{array}$ & $\begin{array}{c}2 \\
150 \\
1 \mathrm{~h} \\
2 \\
2 \mathrm{~h}\end{array}$ & $\begin{array}{l}9,867.63 \\
9,870.87 \\
9,887.4 \\
9,892.99 \\
9,906.1\end{array}$ & $\begin{array}{l}b^{4} \mathrm{P}_{1}-{ }^{6} \mathrm{D}_{2}^{\prime} \\
b^{2} \mathrm{D}_{2}^{\prime}-\alpha^{2} \mathrm{~F}_{3} \\
c^{4} \mathrm{G}_{4}^{\prime}-\left[\mathrm{F}_{4}\right]\end{array}$ \\
\hline $\begin{array}{l}10,078.62 \\
10,052.96 \\
10,048.80 \\
10,046.31 \\
10,031.44\end{array}$ & $\begin{array}{r}100 \\
8 \\
3 \\
150 \\
5\end{array}$ & $\begin{array}{l}9,919.34 \\
9,944.60 \\
9,948.71 \\
9,951.18 \\
9,965.93\end{array}$ & $\begin{array}{l}c^{4} \mathrm{~F}_{5}^{\prime}-\left[{ }^{4} \mathrm{~F}_{5}\right] \\
c^{4} \mathrm{~F}_{5}^{\prime}-\left[\mathrm{H}_{6}\right] \\
b^{2} \mathrm{D}_{3}-a^{2} \mathrm{~F}_{4}^{\prime} \\
b^{4} \mathrm{P}_{2}-{ }^{6} \mathrm{D}_{3}^{\prime}\end{array}$ \\
\hline $\begin{array}{c}10,021.48 \\
10,019.05 \\
10,007.78 \\
9,999.7 \\
9,952.2\end{array}$ & $\begin{array}{c}4 \mathrm{~h} \\
30 \mathrm{~h} \\
3 \\
2 \mathrm{~h} \\
3 \mathrm{~h}\end{array}$ & $\begin{array}{r}9,975.83 \\
9,978.25 \\
9,989.49 \\
9,997.6 \\
10,045.3\end{array}$ & $\begin{array}{l}c^{4} \mathrm{~F}_{5}^{\prime}-\left[{ }^{4} \mathrm{G}_{6},{ }^{4} \mathrm{~F}_{5}\right] \\
b^{2} \mathrm{D}_{2}-b^{4} \mathrm{D}_{2}{ }^{\prime} ? \\
b^{2} \mathrm{~F}_{3}{ }^{\prime}-\alpha^{4} \mathrm{~F}_{2}\end{array}$ \\
\hline $\begin{array}{l}9,940.69 \\
9,918.1 \\
9,912.73 \\
9,909.52 \\
9,890.92\end{array}$ & $\begin{array}{c}2 \\
1 \\
10 \\
1 \mathrm{~h} \\
30\end{array}$ & $\begin{array}{l}10,056.91 \\
10,079.8 \\
10,085.27 \\
10,088.54 \\
10,107.51\end{array}$ & $\begin{array}{c}\left(a^{2} \mathrm{P}_{2}^{\prime}\right)-\left[\mathrm{F}_{3}, \mathrm{D}_{2}\right] \\
b^{4} \mathrm{P}_{3}-\mathrm{D}_{4}^{\prime} \\
b^{2} \mathrm{D}_{3}-b^{4} \mathrm{D}_{4}^{\prime}\end{array}$ \\
\hline
\end{tabular}


TABLE 4.-Wave lengths in the infra-red spectrum of cobalt-Continued

\begin{tabular}{|c|c|c|c|}
\hline$\lambda_{\text {air }}$ I. A. & Intensity & $\nu_{\mathrm{vac}} \mathrm{cm}-1$ & Term combination \\
\hline $\begin{array}{l}9,859.90 \\
9,852.5 \\
9,847.7 \\
9,823.52 \\
9,798.37\end{array}$ & $\begin{array}{l}1 \\
1 \\
2 \\
4 \mathrm{~h} \\
2 \mathrm{~h}\end{array}$ & $\begin{array}{l}10,139.31 \\
10,146.9 \\
10,151.9 \\
10,176.87 \\
10,202.99\end{array}$ & $\begin{array}{l}c^{4} \mathrm{~F}_{4}^{\prime}-\left[\mathrm{F}_{3}\right] \\
c^{4} \mathrm{~F}_{4}^{\prime}-\left[\mathrm{F}_{4}, \mathrm{D}_{3}\right] \\
c^{4} \mathrm{~F}_{4}^{\prime}-\left[\mathrm{F}_{4}, \mathrm{D}_{3}\right]\end{array}$ \\
\hline $\begin{array}{l}9,785.39 \\
9,769.0 \\
9,764.53 \\
9,746.02 \\
9,738.46\end{array}$ & $\begin{array}{c}40 \\
1 \mathrm{~h} \\
5 \mathrm{~h} \\
100 \\
2\end{array}$ & $\begin{array}{l}10,216.52 \\
10,233.7 \\
10,238.34 \\
10,257.79 \\
10,265.78\end{array}$ & \\
\hline $\begin{array}{l}9,735.53 \\
9,729.54 \\
9,695.60 \\
9,694.0 \\
9,678.21\end{array}$ & $\begin{array}{c}2 \\
3 \\
5 \\
2 \\
10 \mathrm{~h}\end{array}$ & $\begin{array}{l}10,268.84 \\
10,275.16 \\
10,310.06 \\
10,312.8 \\
10,329.66\end{array}$ & $b^{2} \mathrm{D}_{2}-a^{2} \mathrm{D}_{3}^{\prime}$ \\
\hline $\begin{array}{l}9,670.20 \\
9,659.94 \\
9,638.21 \\
9,629.83 \\
9,626.72\end{array}$ & $\begin{array}{l}2 \\
3 \\
1 \\
3 \mathrm{~h} \\
1\end{array}$ & $\begin{array}{l}10,338.21 \\
10,349.20 \\
10,372.53 \\
10,381.56 \\
10,384.91\end{array}$ & 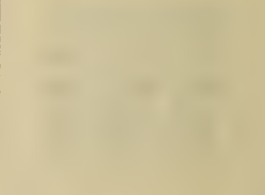 \\
\hline $\begin{array}{l}9,618.32 \\
9,613.46 \\
9,606.52 \\
9,597.90 \\
9,592.3\end{array}$ & $\begin{array}{c}2 \\
4 \\
5 \\
200 \\
2 \mathrm{~h}\end{array}$ & $\begin{array}{l}10,393.98 \\
10,399.23 \\
10,406.75 \\
10,416.09 \\
10,422.2\end{array}$ & $b^{2} \mathrm{~F}_{3}{ }^{\prime}-\alpha^{2} \mathrm{~F}_{3}$ \\
\hline $\begin{array}{l}9,585.28 \\
9,580.63 \\
9,569.00 \\
9,548.66 \\
9,544.52\end{array}$ & $\begin{array}{l}2 \\
3 \\
5 \mathrm{~h} \\
4 \\
300\end{array}$ & $\begin{array}{l}10,429.80 \\
10,434.87 \\
10,447.55 \\
10,469.80 \\
10,474.34\end{array}$ & $\begin{array}{l}c^{4} \mathrm{G}_{5}^{\prime}-\left[\mathrm{F}_{4}\right] \\
b^{2} \mathrm{~F}_{4}^{\prime}-\alpha^{2} \mathrm{~F}_{4}\end{array}$ \\
\hline $\begin{array}{l}9,527.17 \\
9,517.33 \\
9,513.42 \\
9,500.01 \\
9,482.75\end{array}$ & $\begin{array}{c}10 \mathrm{~h} \\
1 \\
1 \\
1 \\
1\end{array}$ & $\begin{array}{l}10,493.42 \\
10,504.27 \\
10,508.59 \\
10,523.42 \\
10,542.57\end{array}$ & 1 \\
\hline $\begin{array}{l}9,470.74 \\
9,454.23 \\
9,442.34 \\
9,435.70 \\
9,428.8\end{array}$ & $\begin{array}{l}2 \\
3 \mathrm{~h} \\
4 \mathrm{~h} \\
3 \\
1\end{array}$ & $\begin{array}{l}10,555.94 \\
10,574.38 \\
10,587.69 \\
10,595.14 \\
10,602.9\end{array}$ & $\begin{array}{c}b^{4} \mathrm{P}_{3}-{ }^{6} \mathrm{D}_{3}{ }^{\prime} \\
c^{4} \mathrm{G}_{5}-\left[{ }^{4} \mathrm{~F}_{5}\right]\end{array}$ \\
\hline $\begin{array}{l}9,425.8 \\
9,422.60 \\
9,406.12 \\
9,395.11 \\
9,356.98\end{array}$ & $\begin{array}{l}1 \\
3 \mathrm{~h} \\
4 \mathrm{~h} \\
2 \\
200\end{array}$ & $\begin{array}{l}10,606.3 \\
10,609.88 \\
10,628.46 \\
10,640.92 \\
10,684.28\end{array}$ & $1+1=1$ \\
\hline $\begin{array}{l}9,351.06 \\
9,347.88 \\
9,344.93 \\
9,340.54 \\
9,319.53\end{array}$ & $\begin{array}{c}3 \\
2 \mathrm{~h} \\
20 \\
3 \mathrm{~h} \\
2\end{array}$ & $\begin{array}{l}10,691.04 \\
10,694.68 \\
10,698.06 \\
10,703.08 \\
10,727.21\end{array}$ & \\
\hline
\end{tabular}


TABLE 4.-Wave lengths in the infra-red spectrum of cobalt-Continued

\begin{tabular}{|c|c|c|c|}
\hline$\lambda_{a}$ ir I. A. & Intensity & $\nu_{\mathrm{Vac}} \mathrm{cm}^{-1}$ & Term combination \\
\hline $9,280.42$ & 5 & $10,772.42$ & $b^{2} \mathrm{~F}_{4}{ }^{\prime}-\alpha^{6} \mathrm{~F}_{5}$ \\
\hline $9,262.5$ & 2 & $10,793.3$ & $b^{2} \mathrm{D}_{2}-b^{4} \mathrm{~F}_{3}^{\prime}$ \\
\hline $9,258.18$ & 4 & $10,798.30$ & $c^{4} \mathrm{~F}_{4}{ }^{\prime}-\left[\mathrm{F}_{4}\right]$ \\
\hline $9,245.60$ & 1 & $10,812.99$ & $b^{2} \mathrm{D}_{3}-a^{2} \mathrm{G}_{4}{ }^{\prime}$ \\
\hline $9,233.64$ & 1 & $10,827.00$ & $a^{2} \mathrm{P}_{2}-a^{4} \mathrm{~F}_{3}^{\prime}$ \\
\hline $9,217.80$ & $2 \mathrm{~h}$ & $10,845.60$ & \\
\hline $\begin{array}{l}9,207.96 \\
9,204.11\end{array}$ & $\frac{1}{5}$ & $\begin{array}{l}10,857.19 \\
10,861.73\end{array}$ & $b^{2} \mathrm{D}_{3}-a^{2} \mathrm{~F}_{3}^{\prime}$ \\
\hline $9,197.29$ & 1 & $10,869.79$ & $c^{4} \mathrm{~F}_{5}{ }^{\prime}-\left[\mathrm{F}_{4} \mathrm{D}_{3}\right]$ \\
\hline $9,185.95$ & 2 & $10,883.21$ & \\
\hline $9,181.75$ & 5 & $10,888.18$ & \\
\hline $9,177.93$ & $\begin{array}{r}20 \\
2\end{array}$ & $\begin{array}{l}10,892.72 \\
10.907 .47\end{array}$ & \\
\hline $9,150.65$ & $2 \mathrm{~h}$ & $10,925.19$ & \\
\hline $9,133.24$ & 6 & $10,946.02$ & $c^{4} \mathrm{~F}_{4}{ }^{\prime}-\left[{ }^{4} \mathrm{~F}_{5}\right]$ \\
\hline $9,130.50$ & 2 & $10,949.30$ & \\
\hline $\begin{array}{l}9,111.64 \\
9,095.37\end{array}$ & 50 & $\begin{array}{l}10,971.96 \\
10,991.58\end{array}$ & \\
\hline $9,071.35$ & $4 h$ & $11,020.69$ & \\
\hline $9,057.29$ & $2 \mathrm{~h}$ & $11,037.80$ & \\
\hline $9,052.44$ & 2 & $11,043.72$ & $b^{2} \mathrm{D}_{2}-b^{4} \mathrm{~F}_{2}^{\prime}$ \\
\hline $\begin{array}{l}9.040 .0 \\
9,037.87\end{array}$ & 50 & $\begin{array}{l}11,058.9 \\
11,061.52\end{array}$ & \\
\hline $8,997.3$ & 1 & $11,111.4$ & \\
\hline $8,986.51$ & 3 & $11,124.74$ & \\
\hline $8,972.89$ & 7 & $11,141.62$ & $h^{4} \mathrm{~F}^{\prime}-2^{4} \mathrm{~F}$ \\
\hline $\begin{array}{l}8,958.37 \\
8,953.72\end{array}$ & $\begin{array}{r}15 \\
2\end{array}$ & $\begin{array}{l}11,159.68 \\
11,165.48\end{array}$ & $b^{4} \mathrm{~F}_{3}{ }^{-}-\alpha^{4} \mathrm{~F}_{4}$ \\
\hline $8,939.14$ & 5 & $11,183.69$ & \\
\hline $8,926.21$ & 50 & $11,199.89$ & $b^{2} \mathrm{D}_{2}-a^{2} \mathrm{D}_{2}^{\prime}$ \\
\hline $8,904.63$ & 30 & $11,227.03$ & \\
\hline $\begin{array}{l}8,892.49 \\
8,888.70\end{array}$ & $\stackrel{1}{S h}$ & $\begin{array}{l}11,242.36 \\
11,247.15\end{array}$ & $c^{4} \mathrm{D}_{2}^{\prime}-\left[\mathrm{F}_{3}\right]$ \\
\hline $8,886.28$ & 2 & $11,250.22$ & \\
\hline $8,878.28$ & 3 & $11,260.35$ & \\
\hline $8,872.59$ & $\frac{1}{8}$ & $11,267.58$ & $c^{4} \mathrm{D}_{2}^{\prime}-\left[\mathrm{F}_{4} \mathrm{D}_{3}\right]$ \\
\hline $8,856.56$ & $\begin{array}{l}8 \\
3\end{array}$ & $\begin{array}{l}11,204.98 \\
11,287.97\end{array}$ & \\
\hline $8,850.70$ & 30 & $11,295.44$ & $b^{2} F_{4}^{\prime}-\alpha^{2} F_{3}$ \\
\hline $8,837.90$ & $4 \mathrm{~h}$ & $11,311.80$ & \\
\hline 8, 835. 21 & 20 & $11,315.25$ & $b^{4} \mathrm{~F}_{4}^{\prime}-\alpha^{4} \mathrm{~F}_{5}$ \\
\hline $\begin{array}{l}8,819.10 \\
8,779.20\end{array}$ & 3 & $\begin{array}{l}11,350.92 \\
11,387.44\end{array}$ & \\
\hline $8,774.71$ & 2 & $11,393.26$ & $c^{4} \mathrm{D}_{4}^{\prime}-\left[{ }^{4} \mathrm{D}_{4},{ }^{4} \mathrm{P}_{3}\right]$ \\
\hline $8,772.04$ & 2 & $11,396.73$ & \\
\hline $\begin{array}{l}8,766.55 \\
8.759 .58\end{array}$ & $\begin{array}{l}4 \mathrm{~h} \\
3\end{array}$ & $\begin{array}{l}11,403.87 \\
11,412.94\end{array}$ & $c^{4} \mathrm{D}_{4}{ }^{\prime}-\left[{ }^{4} \mathrm{D}_{4}\right]$ \\
\hline $8,750.13$ & 60 & $11,425.27$ & $c^{4} \mathrm{~F}_{4}^{\prime}-\left[\mathrm{F}_{3} \mathrm{D}_{2}\right]$ \\
\hline
\end{tabular}


TABLE 4.-Wave lengths in the infra-red spectrum of cobalt-Continued

\begin{tabular}{|l|r|l|l|}
\hline$\lambda_{\text {air }} \mathrm{I} . \mathrm{A}$. & Intensity & $\nu_{\mathrm{va0}} \mathrm{cm}^{-1}$ & Term combination \\
\cline { 1 - 3 } $8,745.56$ & 8 & $11,431.24$ & $b^{4} \mathrm{G}_{3}{ }^{\prime}-\alpha^{4} \mathrm{~F}_{4}$ \\
$8,744.37$ & 10 & $11,432.79$ & \\
$8,733.27$ & 40 & $11,447.32$ & \\
$8,728.5$ & 2 & $11,453.6$ & \\
$8,722.12$ & 2 & $11,461.96$ & \\
$8,678.65$ & 3 & $11,519.37$ & \\
$8,675.02$ & 20 & $11,524.19$ & $a^{2} \mathrm{D}_{2}{ }^{\prime}-\alpha^{4} \mathrm{~F}_{3}$ \\
$8,673.02$ & 2 & $11,526.85$ & \\
$8,661.06$ & 80 & $11,542.76$ & $b^{2} \mathrm{D}_{3}-a^{2} \mathrm{D}_{3}{ }^{\prime}$ \\
$8,658.14$ & 3 & $11,546.66$ & $b^{2} \mathrm{D}_{3}-b^{4} \mathrm{~F}_{4}^{\prime}$ \\
$8,655.73$ & $3 \mathrm{~h}$ & $11,549.85$ & \\
$8,648.79$ & 4 & $11,559.14$ & $a^{2} \mathrm{P}_{2}-a^{4} \mathrm{D}_{3}{ }^{\prime}$ \\
\hline
\end{tabular}

\section{NICKEL}

All of the known and classified lines $(1,071)$ of $\mathrm{Ni}$ I were published by Russell in $1929,{ }^{12}$ but only a dozen lines had been observed photographically beyond $8,700 \mathrm{~A}$. The new results obtained with xenocyanine plates appear in Table 5 , in which the combinations have all been derived from Russell's term tables. The infra-red, as well as other portions of the Ni I spectrum, appears to be less complex than Co I, and relatively simple compared with Fe I. Almost all of the new lines have been accounted for, and the analysis of the Ni I spectrum may be regarded as very nearly completed.

The infra-red arc spectrum of nickel was investigated radiometrically by Randall and Barker, ${ }^{13}$ and in the range 9,520 to $10,980 \mathrm{~A}$ six lines coincide, within the errors of measurement, with the strongest we have recorded photographically. Comparison of our intensity estimates with their galvanometer deflections indicates that the photographic sensitivity of xenocyanine plates for wave length $11,000 \mathrm{~A}$ is approximately 5 per cent that for wave lengths below $10,300 \mathrm{~A}$.

12 H. N. Russell, Phys. Rev., vol. 34, p. 821, 1929.

13 H. M. Randall and E. F. Barker, Astrophys. J., vol. 49, p. 55, 1919. 
TABLE 5.-Wave lengths in the infra-red spectrum of nickel

\begin{tabular}{|c|c|c|c|}
\hline$\lambda_{\mathrm{air}}$ I. A. & Intensity & $\nu_{\mathrm{VAO}} \mathrm{cm}^{-1}$ & Term combination \\
\hline $\begin{array}{l}10,979.87 \\
10,891.25 \\
10,762.24 \\
10,530.53 \\
10,378.62\end{array}$ & $\begin{array}{r}5 \\
2 \\
2 \\
20 \\
100\end{array}$ & $\begin{array}{l}9,105.09 \\
9,179.17 \\
9,289.20 \\
9,493.60 \\
9,632.56\end{array}$ & $\begin{array}{l}y^{3} \mathrm{D}_{3}^{\circ}-e^{3} \mathrm{D}_{3} \\
y^{3} \mathbf{F}_{2}^{0}-e^{3} \mathrm{D}_{2} \\
y^{3} \mathrm{D}_{3}^{\circ}-e^{3} \mathrm{D}_{2} \\
y^{3} \mathbf{F}_{3}^{\circ}-e^{3} \mathrm{D}_{3} \\
y^{3} \mathbf{F}_{4}^{\circ}-e^{3} \mathbf{D}_{3}\end{array}$ \\
\hline $\begin{array}{l}10,330.23 \\
10,321.10 \\
10,302.61 \\
10,295.05 \\
10,226.15\end{array}$ & $\begin{array}{r}50 \\
5 \\
50 \\
5 \\
4\end{array}$ & $\begin{array}{l}9,677.68 \\
9,686.23 \\
9,703.62 \\
9,710.75 \\
9,776.18\end{array}$ & $\begin{array}{c}y^{3} \mathrm{~F}_{3}^{\circ}-e^{3} \mathrm{D}_{2} \\
w^{3} \mathrm{~F}_{3}^{\circ}-g^{3} \mathrm{~F}_{3} \\
y^{3} \mathrm{D}_{1}^{\circ}-e^{3} \mathrm{D}_{1} \\
\\
w^{3} \mathrm{D}_{2}^{\circ}-g^{3} \mathrm{~F}_{3}\end{array}$ \\
\hline $\begin{array}{r}10,193.25 \\
10,145.37 \\
10,061.29 \\
10,048.60 \\
9,898.90\end{array}$ & $\begin{array}{r}100 \\
20 \\
10 \\
10 \\
40\end{array}$ & $\begin{array}{r}9,807.73 \\
9,854.01 \\
9,936.36 \\
9,948.91 \\
10,099.36\end{array}$ & $\begin{array}{l}z^{1} \mathrm{P}_{1}^{\circ}-e^{3} \mathrm{D}_{2} \\
y^{3} \mathrm{D}_{1}^{\circ}-e^{1} \mathrm{D}_{2} \\
y^{3} \mathrm{G}_{4}^{\circ}-g^{3} \mathrm{~F}_{3} \\
y^{3} \mathrm{D}_{2}^{\circ}-e^{3} \mathrm{D}_{1} \\
y^{3} \mathrm{D}_{2}^{0}-e^{1} \mathrm{D}_{2}\end{array}$ \\
\hline $\begin{array}{l}9,842.04 \\
9,710.1 \\
9,689.35 \\
9,520.06 \\
9,447.29\end{array}$ & $\begin{array}{r}2 \\
1 \\
3 \\
100 \\
5\end{array}$ & $\begin{array}{l}10,157.71 \\
10,295.74 \\
10,317.78 \\
10,501.26 \\
10,582.15\end{array}$ & $\begin{array}{l}y^{3} \mathrm{D}_{3}^{\circ}-f^{1} \mathrm{~F}_{3} \\
x^{1} \mathrm{D}_{2}^{\circ}-g^{3} \mathrm{~F}_{3} \\
y^{3} \mathrm{~F}_{2}^{\circ}-e^{3} \mathrm{D}_{1} \\
x^{3} \mathrm{~F}_{3}^{\circ}-g^{3} \mathrm{~F}_{4}\end{array}$ \\
\hline $\begin{array}{l}9,396.57 \\
9,385.62 \\
9,258.47 \\
9,196.18 \\
9,106.40\end{array}$ & $\begin{array}{r}20 \\
10 \\
5 \\
2 \\
30\end{array}$ & $\begin{array}{l}10,639.26 \\
10,651.68 \\
10,797.96 \\
10,871.10 \\
10,978.28\end{array}$ & $\begin{array}{c}y^{3} \mathrm{~F}_{2}^{\circ}-e^{1} \mathrm{D}_{2} \\
a^{1} \mathrm{G}_{4}-y^{3} \mathrm{~F}_{4}^{\circ} \\
w^{3} \mathrm{~F}_{4}-g^{3} \mathrm{~F}_{4}\end{array}$ \\
\hline $\begin{array}{l}9,085.25 \\
9,078.70 \\
9,058.56 \\
9,005.14 \\
8,982.35\end{array}$ & $\begin{array}{r}3 \\
10 \\
6 \\
5 \\
2\end{array}$ & $\begin{array}{l}11,003.84 \\
11,011.77 \\
11,036.25 \\
11,101.72 \\
11,129.89\end{array}$ & $\begin{array}{l}z^{3} \mathrm{G}_{3}^{\circ}-e^{3} \mathrm{D}_{2} \\
w^{3} \mathrm{~F}_{3}^{\circ}-f^{1} \mathrm{~F}_{3} \\
\\
w^{3} \mathrm{D}_{2}^{\circ}-f^{1} \mathrm{~F}_{3} \\
z^{1} \mathrm{P}_{1}^{\circ}-e^{3} \mathrm{D}_{1}\end{array}$ \\
\hline $\begin{array}{l}8,968.20 \\
8,965.94 \\
8,954.65 \\
8,877.07 \\
8,862.59\end{array}$ & $\begin{array}{r}30 \\
50 \\
2 \\
10 \\
100\end{array}$ & $\begin{array}{l}11,147.45 \\
11,150.26 \\
11,164.32 \\
11,261.89 \\
11,280.29\end{array}$ & $\begin{array}{c}y^{3} \mathrm{G}_{5}^{\circ}-g^{3} \mathrm{~F}_{4} \\
y^{3} \mathrm{~F}_{3}^{\circ}-e^{1} \mathrm{D}_{2} \\
z^{1} \mathrm{D}_{2}^{\circ}-e^{3} \mathrm{D}_{3} \\
y^{3} \mathrm{G}_{4}^{\circ}-f^{1} \mathrm{~F}_{3} \\
z^{1} \mathrm{P}_{1}^{\circ}-e^{1} \mathrm{D}_{2}\end{array}$ \\
\hline $\begin{array}{l}8,824.2 \\
8,809.47 \\
8,770.68 \\
8,716.58 \\
8,706.05\end{array}$ & $\begin{array}{r}1 \\
30 \\
10 \\
3 \\
1\end{array}$ & $\begin{array}{l}11,329.4 \\
11,348.30 \\
11,398.50 \\
11,469.24 \\
11,483.12\end{array}$ & $\begin{array}{l}z^{1} \mathrm{D}_{2}^{\circ}-e^{3} \mathrm{D}_{2} \\
a^{1} \mathrm{G}_{4}-y^{3} \mathrm{D}_{3}^{\circ} \\
w^{3} \mathrm{D}_{3}^{\circ}-g^{3} \mathrm{~F}_{4}\end{array}$ \\
\hline $\begin{array}{l}8,702.49 \\
8,688.64 \\
8,680.2 \\
8,661.85 \\
8,637.04\end{array}$ & $\begin{array}{r}6 \\
3 \\
1 \\
2 \\
15\end{array}$ & $\begin{array}{l}11,487.81 \\
11,506.12 \\
11,517.3 \\
11,541.71 \\
11,574.86\end{array}$ & $z^{1} \mathrm{~F}_{3}^{\circ}-e^{3} \mathrm{D}_{3}$ \\
\hline $\begin{array}{l}8,606.45 \\
8,598.84 \\
8,586.2 \\
8,580.04\end{array}$ & $\begin{array}{r}10 \\
2 \\
1 \\
2\end{array}$ & $\begin{array}{l}11,616.00 \\
11,626.29 \\
11,643.40 \\
11,651.76\end{array}$ & $\begin{array}{l}x^{3} \mathrm{D}_{3}^{\circ}-g^{3} \mathrm{~F}_{4} \\
z^{3} \mathrm{G}_{4}^{\circ}-e^{3} \mathrm{D}_{3} \\
x^{1} \mathrm{D}_{2}^{\circ}-f^{1} \mathrm{~F}_{3} \\
y^{3} \mathrm{G}_{5}^{\circ}-h^{3} \mathrm{~F}_{4}\end{array}$ \\
\hline
\end{tabular}




\section{ZIRCONIUM}

About a year ago Kiess and Kiess ${ }^{14}$ published an analysis of $\mathrm{Zr}$ I based on a list of approximately 1,600 wave lengths measured at the Bureau of Standards. To this list, which terminated at 9,276 A, we are now able to add the lines given in Table 6 . All of the strong lines and many of the fainter ones are accounted for as combinations between the terms given in the paper cited above. No new terms have been revealed by these supplementary observations, although several metastable terms arising from the configuration $4 d^{4}$ are still to be found.

A conspicuous feature of the infra-red $\mathrm{Zr}$ spectrum is a group of bands, shaded toward longer wave lengths, lying between 9,300 and $9,500 \mathrm{~A}$. No regularities have been detected among these bands that will relate them to the systems recently described by Miss Lowater. ${ }^{15}$ The wave lengths, estimated intensities, and wave numbers of the heads of these bands are given in Table 7 .

TABLE 6.-Wave lengths in the infra-red spectrum of zirconium

\begin{tabular}{|c|c|c|c|}
\hline$\lambda_{\text {air }}$ I. A. & Intensity & $\nu_{\mathrm{PaC}} \mathrm{cm}^{-1}$ & Term combination \\
\hline $\begin{array}{l}10,738.94 \\
10,696.67 \\
10,654.11 \\
10,551.46 \\
10,515.86\end{array}$ & $\begin{array}{l}1 \\
1 \\
1 \\
1 \\
1\end{array}$ & $\begin{array}{l}9,309.36 \\
9,346.14 \\
9,383.48 \\
9,474.77 \\
9,506.84\end{array}$ & $\begin{array}{l}a^{3} \mathrm{D}_{3}-y^{3} \mathrm{~F}_{4}^{\circ} \\
a^{3} \mathrm{G}_{3}-z^{3} \mathrm{G}_{3}^{\circ} \\
a^{3} \mathrm{G}_{4}-z^{3} \mathrm{G}_{4}^{\circ} \\
a^{3} \mathrm{D}_{1}-y^{3} \mathrm{~F}_{2}^{\circ} \\
b^{3} \mathrm{~F}_{4}-z^{3} \mathrm{G}_{3}^{\circ}\end{array}$ \\
\hline $\begin{array}{l}10,433.73 \\
10,328.41 \\
10,242.85 \\
10,210.44 \\
10,199.42\end{array}$ & $\begin{array}{r}1 \\
1 \\
2 \\
10 \\
4\end{array}$ & $\begin{array}{l}9,581.67 \\
9,679.38 \\
9,760.24 \\
9,791.21 \\
9,801.80\end{array}$ & $\begin{array}{l}a^{3} \mathrm{D}_{3}-y^{5} \mathrm{D}_{4}^{\circ} \\
a^{5} \mathrm{~F}_{2}-z^{5} \mathrm{G}_{2}^{\circ} \\
a^{3} \mathrm{G}_{5}-z^{3} \mathrm{G}_{5}^{\circ} \\
b^{3} \mathrm{~F}_{4}-z^{3} \mathrm{G}_{4}^{\circ}\end{array}$ \\
\hline $\begin{array}{l}10,105.30 \\
10,084.70 \\
10,045.15 \\
10,028.68 \\
10,016.97\end{array}$ & $\begin{array}{r}1 \\
12 \\
3 \\
2 \\
2\end{array}$ & $\begin{array}{l}9,893.08 \\
9,913.30 \\
9,952.33 \\
9,968.67 \\
9,980.33\end{array}$ & $\begin{array}{l}b^{3} \mathrm{~F}_{3}-z^{3} \mathrm{G}_{3}^{\circ} \\
a^{5} \mathrm{~F}_{1}-z^{5} \mathrm{G}_{2}^{\circ} \\
a^{5} \mathrm{~F}_{3}-z^{5} \mathrm{G}_{3}^{\circ} \\
c^{3} \mathrm{P}_{2}-x^{3} \mathrm{D}_{3}^{\circ}\end{array}$ \\
\hline $\begin{array}{l}9,990.44 \\
9,958.58 \\
9,928.53 \\
9,909.76 \\
9,822.30\end{array}$ & $\begin{array}{r}1 \\
2 \\
1 \\
3 \\
20\end{array}$ & $\begin{array}{l}10,006.83 \\
10,038.84 \\
10,069.22 \\
10,088.30 \\
10,178.12\end{array}$ & $a^{5} \mathrm{~F}_{2}-z^{5} \mathrm{G}_{3}^{\circ}$ \\
\hline $\begin{array}{l}9,820.42 \\
9,812.85 \\
9,792.74 \\
9,780.40 \\
9,773.30\end{array}$ & $\begin{array}{r}3 \\
10 \\
6 \\
18 \\
0\end{array}$ & $\begin{array}{l}10,180.07 \\
10,187.93 \\
10,208.85 \\
10,221.73 \\
10,229.15\end{array}$ & $\begin{array}{l}a^{5} \mathrm{~F}_{4}-z^{5} \mathrm{G}_{4} \\
b^{3} \mathrm{~F}_{3}-z^{3} \mathrm{G}_{4}^{\circ} \\
b^{3} \mathrm{~F}_{2}-z^{3} \mathrm{G}_{3}^{\circ} \\
b^{3} \mathrm{~F}_{4}-z^{3} \mathrm{G}_{5}^{\circ}\end{array}$ \\
\hline $\begin{array}{l}9,666.82 \\
9,547.26 \\
9,493.47 \\
9,483.35 \\
9,441.26\end{array}$ & $\begin{array}{r}1 \\
25 \\
1 \\
3 \\
1\end{array}$ & $\begin{array}{l}10,341.83 \\
10,471.34 \\
10,530.67 \\
10,541.91 \\
10,588.90 \\
\end{array}$ & $\begin{array}{l}a^{5} \mathrm{~F}_{3}-z^{5} \mathrm{G}_{4} \\
c^{3} \mathrm{P}_{2}-y^{3} \mathrm{P}_{2}^{\circ}\end{array}$ \\
\hline
\end{tabular}

11 B. S. Jour. Research (RP296), vol. 6, p. 621, 1931.

15 Proc. Phys. Soc. (London), vol. 44, p. 51, 1932. 
TABLE 6.-Wave lengths in the infra-red spectrum of zirconium-Continued

\begin{tabular}{|c|c|c|c|}
\hline$\lambda_{\text {air I. A. }}$ & Intensity & $\nu_{\mathrm{VBO}} \mathrm{cm}^{-1}$ & Term combination \\
\hline $9,438.35$ & 1 & $10,592.17$ & \multirow{5}{*}{$c^{3} \mathrm{P}_{1}-y^{3} \mathrm{P}_{2}^{\circ}$} \\
\hline $9,419.36$ & 1 & $10,613.53$ & \\
\hline $9,405.00$ & 2 & $10,629.73$ & \\
\hline $9,358.32$ & 2 & $10,682.74$ & \\
\hline $9,352.43$ & 1 & $10,689.48$ & \\
\hline $9,318.19$ & 4 & $10,728.76$ & \multirow[b]{3}{*}{$\begin{array}{l}a^{5} \mathrm{~F}_{4}-z^{5} \mathrm{G}_{5}^{\circ} \\
a^{3} \mathrm{G}_{4}-y^{3} \mathrm{~F}_{3}^{\circ}\end{array}$} \\
\hline $9,290.30$ & 0 & $10,760.96$ & \\
\hline $\begin{array}{l}9,276.89 \\
9,251.17\end{array}$ & $\begin{array}{r}25 \\
5\end{array}$ & $\begin{array}{l}10,776.52 \\
10,806.48\end{array}$ & \\
\hline $9.242,65$ & 5 & 10.816 .44 & \multirow{2}{*}{$\begin{array}{l}c^{3} \mathrm{P}_{1}-w^{3} \mathrm{~F}_{2}^{\circ} \\
a^{3} \mathrm{G}_{3}-y^{3} \mathrm{D}_{2}^{\circ}\end{array}$} \\
\hline $9.229,33$ & 3 & $10.832,05$ & \\
\hline $9,182.30$ & 0 & $10,887.53$ & \multirow[b]{2}{*}{$a^{3} \mathrm{G}_{4}-y^{3} \mathrm{D}_{3}^{\circ}$} \\
\hline $9,171.50$ & 3 & $10,900.35$ & \\
\hline $9,140.40$ & 0 & $10,937.44$ & \multirow{4}{*}{$\begin{array}{c}a^{3} \mathrm{H}_{4}-y^{3} \mathrm{G}_{3}^{\circ} \\
\left\{\begin{array}{l}c^{3} \mathrm{~F}_{3}-x^{3} \mathrm{~F}_{3}^{\circ} \\
a^{3} \mathrm{G}_{3}-y^{5} \mathrm{D}_{2}^{\circ}\end{array}\right.\end{array}$} \\
\hline $9,139.36$ & 10 & $10,938.69$ & \\
\hline $9,134.23$ & 1 & $10,944.83$ & \\
\hline $9,099.90$ & 2 & $10,986.12$ & \\
\hline $9,069.41$ & 15 & $11,023.05$ & \multirow{4}{*}{$\begin{array}{l}a^{3} \mathrm{H}_{5}-y^{3} \mathrm{G}_{4}^{\circ} \\
a^{5} \mathrm{~F}_{5}-z^{5} \mathrm{G}_{6}^{\circ} \\
a^{3} \mathrm{G}_{3}-y^{3} \mathrm{~F}_{2}^{\circ} \\
c^{3} \mathrm{~F}_{4}-x^{3} \mathrm{~F}_{4}^{\circ}\end{array}$} \\
\hline $9,015.16$ & 20 & $11,089.39$ & \\
\hline $9,011.34$ & 2 & $11,094.09$ & \\
\hline $8,975.78$ & 0 & $11,138.05$ & \\
\hline
\end{tabular}

TABLE 7.-Infra-red zirconium bands

\begin{tabular}{|c|c|c|c|c|c|}
\hline$\lambda_{\text {air I. A. }}$ & Intensity & $\nu_{\mathrm{VBO}} \mathrm{cm}^{-1}$ & $\lambda_{\text {air }}$ I. A. & Intensity & $\nu_{\mathrm{vac}} \mathrm{cm}^{-1}$ \\
\hline $\begin{array}{l}9,483.55 \\
9,476.65 \\
9,453.55 \\
9,443.93 \\
9,438.24\end{array}$ & $\begin{array}{l}2 \\
1 \\
1 \\
1 \\
1\end{array}$ & $\begin{array}{l}10,541.69 \\
10,549.36 \\
10,575.14 \\
10,585.91 \\
10,592.29\end{array}$ & $\begin{array}{l}9,397.21 \\
9,387.26 \\
9,370.74 \\
9,350.34 \\
9,358.33\end{array}$ & $\begin{array}{l}2 \\
2 \\
3 \\
3 \\
1 \\
1\end{array}$ & $\begin{array}{l}10,638.54 \\
10,649.82 \\
10,668.59 \\
10,680.45 \\
10,682.74\end{array}$ \\
\hline $\begin{array}{l}9,431.75 \\
9,412.03 \\
9,408.92 \\
9,402.84 \\
9,401.09\end{array}$ & $\begin{array}{l}2 \\
2 \\
1 \\
2 \\
2\end{array}$ & $\begin{array}{l}10,599.58 \\
10,621.79 \\
10,625.30 \\
10,632.17 \\
10,634.15\end{array}$ & $\begin{array}{l}9,356.12 \\
9,343.19 \\
9,329.93 \\
9,315.87 \\
9,299.56\end{array}$ & $\begin{array}{l}3 \\
4 \\
5 \\
5 \\
5\end{array}$ & $\begin{array}{l}10,685.26 \\
10,700.05 \\
10,715.26 \\
10,731.43 \\
10,750.25\end{array}$ \\
\hline
\end{tabular}

\section{CONCLUSION}

The data presented in the tables of this paper furnish ample proof of the effectiveness of xenocyanine in bringing into the domain of precise measurement a region of the spectrum heretofore accessible only with radiometric devices. Their application to the investigation of the structure of near-infra-red band spectra, a problem in which great efforts are being made to secure the advantages of increased resolving power, is obvious. In astrophysics the identification of unknown lines in the spectra of the sun and stars is advanced by each extension of our knowledge of the emission spectra of the elements. Reference to the Revision of Rowland's Preliminary Table of Solar

$$
132919-32-4
$$


Spectrum Wave-Lengths ${ }^{16}$ will show that several of the unidentified infra-red solar lines are due to the five elements whose spectra are described above. Both $\mathrm{Ti}$ and $\mathrm{Fe}$ are rich in strong lines in the region from 9,000 to nearly $11,000 \mathrm{~A}$ and may be used to supply comparison spectra for observations made with dispersing systems other than concave gratings. However, the values of the $\mathrm{Fe}$ wave lengths to be used as standards of reference may be calculated from the terms determined by interference methods more accurately than the observed values given in Table 3.

As stated above, we have completed a survey of the infra-red spectra of about 50 elements. We shall present the new wave lengths in subsequent papers in this Journal as soon as the data have been compiled for publication.

Washington, June 21, 1932.

16 Carnegie Institution of Washington, Publ. No. 396, 1928. 